ARTICLE

\title{
Antibiotic-chemoattractants enhance neutrophil clearance of Staphylococcus aureus
}

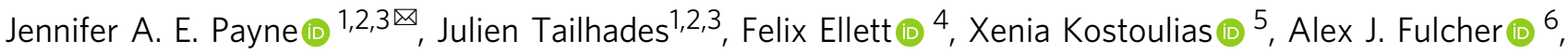

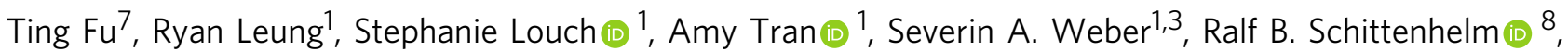 \\ Graham J. Lieschke (1D 9 ${ }^{9}$, Chengxue Helena Qin (10) 7,10, Daniel Irima (1D ${ }^{4}$, Anton Y. Peleg (1D ${ }^{5,11,12}$ \& \\ Max J. Cryle (iD 1,2,3凶
}

The pathogen Staphylococcus aureus can readily develop antibiotic resistance and evade the human immune system, which is associated with reduced levels of neutrophil recruitment. Here, we present a class of antibacterial peptides with potential to act both as antibiotics and as neutrophil chemoattractants. The compounds, which we term 'antibiotic-chemoattractants', consist of a formylated peptide (known to act as chemoattractant for neutrophil recruitment) that is covalently linked to the antibiotic vancomycin (known to bind to the bacterial cell wall). We use a combination of in vitro assays, cellular assays, infection-on-achip and in vivo mouse models to show that the compounds improve the recruitment, engulfment and killing of $S$. aureus by neutrophils. Furthermore, optimizing the formyl peptide sequence can enhance neutrophil activity through differential activation of formyl peptide receptors. Thus, we propose antibiotic-chemoattractants as an alternate approach for antibiotic development.

\footnotetext{
${ }^{1}$ Infection and Immunity Program, Monash Biomedicine Discovery Institute, Department of Biochemistry and Molecular Biology, Monash University, Clayton, Victoria 3800, Australia. ${ }^{2}$ ARC Centre of Excellence for Innovations in Peptide and Protein Science, Monash University, Clayton, Victoria 3800, Australia. ${ }^{3}$ EMBL Australia, Monash University, Clayton, Victoria 3800, Australia. ${ }^{4}$ BioMEMS Resource Center, Center for Engineering in Medicine and Surgical Services, Massachusetts General Hospital, Shriners Hospital for Children, and Harvard Medical School, Charlestown, MA 02129, USA. ${ }^{5}$ Infection and Immunity Program, Monash Biomedicine Discovery Institute and Department of Microbiology, Monash University, Clayton, Victoria 3800, Australia. ${ }^{6}$ Monash Micro Imaging, Monash University, Clayton, Victoria 3800, Australia. ${ }^{7}$ Drug Discovery Biology, Monash Institute of Pharmaceutical Sciences, Monash University, Parkville, Victoria 3052, Australia. ${ }^{8}$ Monash Proteomics and Metabolomics Facility, Monash University, Clayton, Victoria 3800, Australia. ${ }^{9}$ Australian Regenerative Medicine Institute, Monash University, Clayton, Victoria 3800, Australia. ${ }^{10}$ Baker Heart and Diabetes Institute, Melbourne, Victoria 3004, Australia. ${ }^{11}$ Department of Infectious Diseases, The Alfred Hospital, Melbourne, Victoria 3004, Australia. ${ }^{12}$ Central Clinical

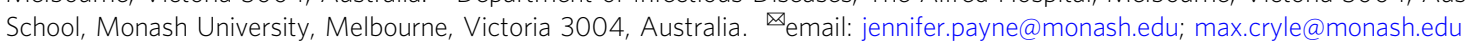


A ntibiotics have become a cornerstone of modern medicine, allowing the prevention and treatment of once deadly infections. However, their durability is hampered by the emergence of bacterial resistance ${ }^{1,2}$. This is particularly notable for the Gram-positive bacteria Staphylococcus aureus where the rise of methicillin resistance (MRSA) has led to an increased reliance on last-resort antibiotics. MRSA is widespread, accounting for $37 \%$ of $S$. aureus bloodstream infections and also has higher mortality rates ${ }^{3}$. In addition to developing antibiotic resistance, $S$. aureus also employs an arsenal of secreted factors to evade the host immune system ${ }^{4}$. Avoiding detection by cells of our innate immune response ensures that first-line responders, such as neutrophils cannot come into play to protect us from this infection ${ }^{4}$. Additionally, resistance to clinical antibiotics, such as methicillin, in $S$. aureus is leading to the emergence of strains with greater immune evasion ${ }^{5,6}$. The increased difficulty in eradicating these strains, therefore, leads to persistent and deadly infections ${ }^{6}$. Understanding the critical need for the development of new antibiotics, the World Health Organization has listed MRSA as a high priority target.

New approaches to tackle this problem are urgently required, which do not rely on the increasingly risky strategy of identifying new antibiotics from nature (the source of the vast majority of clinical agents ${ }^{7}$ ) or increasing the toxicity of the antibiotic. Designer immunotherapeutics to target bacterial infections are one example of a new strategy that is being explored to fill this innovation gap $^{8,9}$. Our body's own immune system provides inspiration and is central to the efficacy of such strategies, with one example being self-assembling peptide nanonets that entrap bacteria and kill them with embedded antimicrobials, taking their inspiration from neutrophil extracellular traps ${ }^{10}$. Another approach is mimicking the body's own host defense peptides that combine two functions in one-direct antimicrobial activity and immune modulation or enhancement ${ }^{11,12}$. The ability of antibiotic-resistant bacteria to avoid immune detection can be overcome using these dual-acting molecules by enhancing the chemoattractant gradient around the bacteria. The establishment of this gradient is an essential step for the recruitment of innate immune cells, such as neutrophils, for prevention, and resolution of infection.

One of the main pathogen-associated chemoattractants are formylated peptides (fPeps). These fPeps are short peptides (3-5 amino acids) that activate innate immune cells by binding to formyl peptide receptors (FPR), stimulating chemotaxis and phagocytosis of the bacteria ${ }^{13,14}$. The importance of fPeps and FPR activation for preventing infection is highlighted by the diversity of proteins that $S$. aureus produces to counter both chemotaxis and FPR activation ${ }^{4}$. These fPeps are the N-terminal fragments of bacterial proteins, as formyl methionine initiates protein biosynthesis in bacteria ${ }^{13,15}$. These bacterially derived peptides are strong inducers of chemotaxis and are necessary for neutrophils to mount a successful immune response; host-derived chemoattractants alone often insufficient for this task ${ }^{16,17}$. This chemoattractant gradient can be enhanced by causing the bacteria to generate more fPeps, as seen in the mechanism of action of actinion, which inhibits bacterial peptide deformylase leading to enhanced release of fPeps from the bacteria ${ }^{18}$. Another strategy being pursued is through artificially enhancing the chemotactic gradient by targeting and attaching chemoattractants, like fPeps, directly to the bacteria. Treatment with an isolated chemotactic agent would be insufficient to generate the gradient required for neutrophil chemotaxis. To provide specificity of immune activation toward the infecting bacteria, a chemotactic agent combined with a bacterial targeting component is necessary.

In the current study, we propose an approach to exploit the properties of fPep chemoattractants by linking them to the clinical antibiotic vancomycin ${ }^{19,20}$, thereby producing an immunotherapeutic to treat $S$. aureus infections (Fig. 1). For these vancomycin-fPep conjugates, vancomycin acts as a targeting agent that binds to the $S$. aureus cell wall. The attached fPep then generates a chemoattractant gradient surrounding $S$. aureus cells, improving the recruitment of neutrophils to the site of infection and enhancing phagocytosis of the bacteria ${ }^{14}$. Aspects of linking a fPep to a targeting element have been examined for Gramnegative bacteria using polymyxins ${ }^{21}$ and for fungi through use of caspofungin or amphotericin $\mathrm{B}^{22}$. However, these studies did not optimize the fPep sequence, which we have found to be essential in order to establishing enhanced activity in these conjugates. The creation of these proof-of-principle antibiotic-chemoattractants required the development of an analytical platform to assess these dual-functional antimicrobial agents that extend beyond the standard assessment of antibiotic action. Through the use of infection-on-a-chip technology, we directly demonstrated that our conjugates modulate the interactions between $S$. aureus and neutrophils by enhancing neutrophil recruitment, phagocytosis, and eventual clearance of $S$. aureus cells. This also proved to be an effective strategy in vivo, where antibiotic-chemoattractants demonstrated reduced bacterial load and improved lung pathology in a mouse MRSA pneumonia model. Taken together, our work demonstrates the promise of possible antibiotic therapy resulting from the targeted activation of the human immune system toward microbes.

\section{Results}

The antibiotic-chemoattractant binds to the surface of antibiotic-resistant $S$. aureus. Vancomycin was chosen as the optimal candidate for our antibiotic-chemoattractant approach, as its mechanism of action involves binding to the bacterial surface ${ }^{19,20}$. An initial fPep (B1, fMLFK(BODIPY)-Pra- $\mathrm{NH}_{2}$ ) was generated by reacting the fPep (R11) with the commercially available BODIPY-OSu ester. Click chemistry was used to generate the C-terminal BODIPY-labelled vancomycin (B2) and the C-terminal BODIPY-labelled fPep-vancomycin conjugate (B3) ${ }^{23,24}$. Using super-resolution confocal microscopy, binding of the labelled antibiotic-fPep conjugate was observed to have a similar binding distribution as the labelled vancomycin alone to MSSA and MRSA strains. This correlates with the findings of previous studies investigating labelled glycopeptide antibiotic (GPA) binding 24-26, with binding to the septum in S. aureus also similar for vancomycin and the conjugate (Supplementary Fig. 1). Conjugate binding was also observed to $B$. subtilis, suggesting the potential for antibiotic-chemoattracts to be used against Grampositive bacteria more generally; no binding was observed to E. coli (B1, Supplementary Fig. 2). Despite binding that followed the same general trends as vancomycin, the antibioticchemoattractant displayed a 5-7-fold increase in the minimal inhibitory concentration against 9 clinical strains of hospitalacquired MRSA and MSSA (Supplementary Table 1). Given this, we next explored the optimal attachment site on vancomycin for the chemoattractant cargo in order to ameliorate this reduction in activity.

Determining the optimum site and linker length for conjugation to vancomycin. We investigated three possible sites of attachment to vancomycin to minimize the reduction in antimicrobial activity: the primary amine on the sugar (V linked); the $\mathrm{N}$-methylated amine (N-linked); or the carboxyl group (C-linked, Fig. 1). We also explored altering the linker length between the fPep and vancomycin by varying the number of flexible and polar polyethylene glycol (PEG) spacer units $(0,3$, or 6 repeats). These fPeps with linkers were prepared by solid-phase peptide synthesis. 

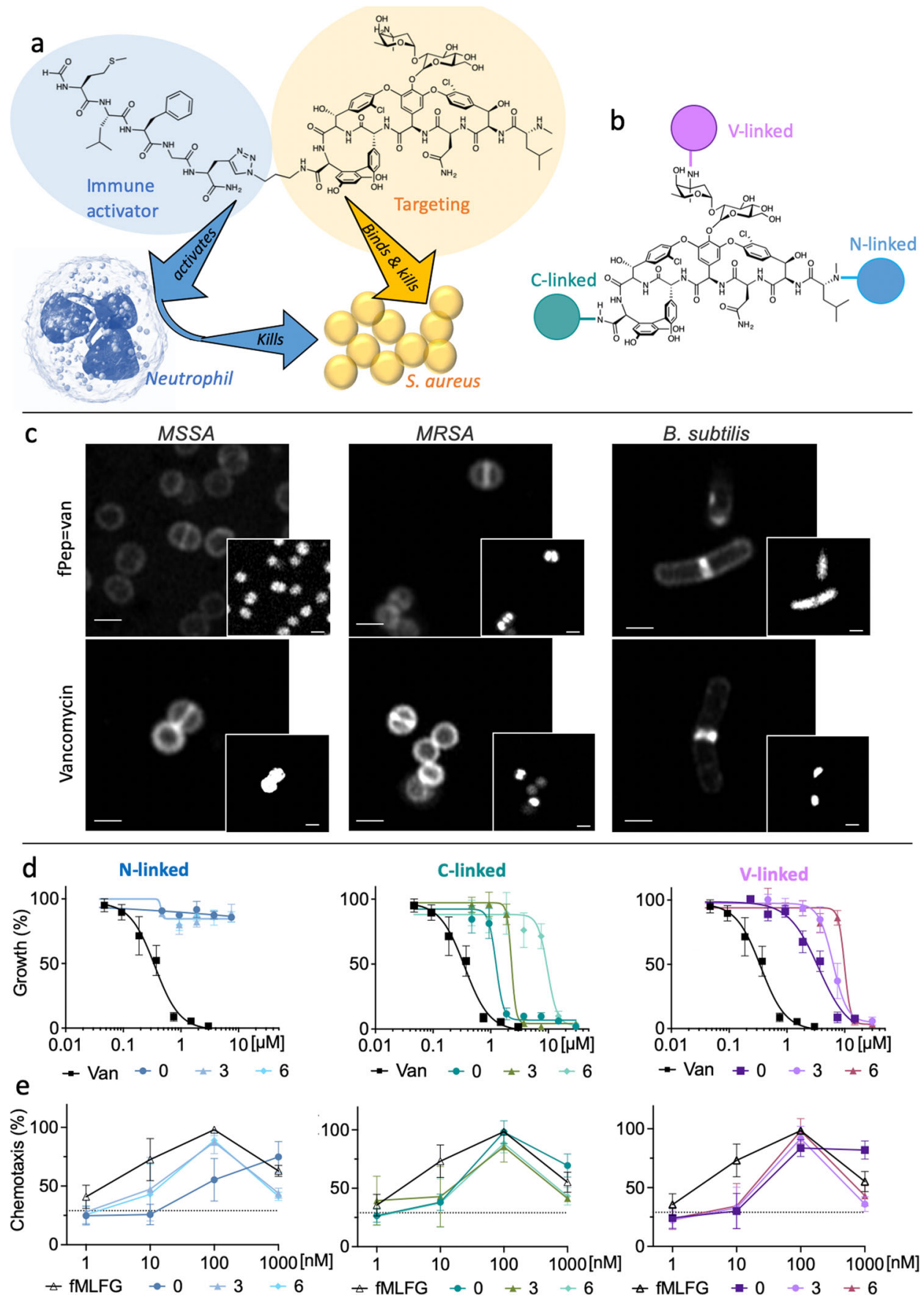

Fig. 1 Overcoming S. aureus immune evasion with immunotherapeutic antibiotics that bind to bacteria, retains activity against bacteria and can recruit neutrophils. a Our strategy combines the possibility of direct killing of the bacteria with enhancing the neutrophil response. Vancomycin (yellow) targets and binds directly to $S$. aureus cell wall, while the fPep helps the immune response (blue) by enhancing the chemotactic gradient, directing immune cells to the bacterial invader and improving phagocytosis. Neutrophil image modified from ${ }^{70}$. b Linkage to vancomycin can be made through three main sites: the vancosamine primary amine (V linked, purple); methylated amine ( $\mathrm{N}$-linked, blue); or the carboxyl group (C-linked, green). c Fluorescently labelled BODIPY vancomycin (B2) or the conjugate ( $f P e p=v a n, B 3$ ) imaged by Airyscan super-resolution microscopy localized to the wall of the Gram-positive bacteria $B$. subtilis, S. aureus-MSSA, and an MRSA clinical isolate. Scale bars are $1 \mu \mathrm{m}$; Insert image is nuclear staining by Hoechst 33342 , images are representative of 3 biological replicates. The linkage site to vancomycin and the length of the PEG linker was critical to maintain antimicrobial activity against $S$. aureus as seen in a microbroth dilution assay (d) but had little effect on the chemotaxis of human neutrophils in transwell assay (e). Three different length PEG linkers were trialed: no linker (0), 3 PEG units (3), or 6 PEG units (6) at the three attachment sites on vancomycin, leading to three different C-linked (C1-3), N-linked (N4-6), and V-linked (V7-9) compounds. The growth of a clinical isolate of S. aureus-MRSA was determined relative to the no protein control. Chemotaxis was calculated relative to the no protein control and $100 \%$ chemotaxis set as the neutrophil recruitment observed to fMLFG (FP1) at $100 \mathrm{nM}$ for each donor. Error bars are SEM, $n=3$ biologically independent experiments. 
Incorporation of a C-terminal L-propargylglycine residue (containing an alkyne group) allowed for the simple conjugation of the fPep to the three different azide-modified vancomycin scaffolds (GPA2, 3, and 4) using copper-catalyzed clickchemistry ${ }^{10,27}$. Monitoring neutrophil chemotaxis via transwell assays demonstrated that the attachment site and length of the linker did not considerably affect human neutrophil recruitment, with the typical bell-shaped profile for chemotaxis agonists noted for all conjugates. The highest levels of recruitment were observed at $100 \mathrm{nM}$ for all attachment sites and linker lengths, similar to the recruitment exhibited by free fMLFG (FP1, Fig. 1). However, at $10 \mathrm{nM}$ there was a decrease in recruitment for all antibioticchemoattractants compared to the AMLG (FP1) alone, indicating possible alterations to the fPep chemotaxis profile due to conjugation to vancomycin.

In contrast to neutrophil recruitment, the site of attachment and a minimal linker length was key to maintaining direct growth inhibition of $S$. aureus strains (Fig. 1), which was important to assess as a proxy for bacterial binding. Activity was tested in a microbroth dilution assay against an MRSA strain (A8090). $\mathrm{N}$-linked compounds (N4-6) were unable to inhibit MRSA growth at the highest concentration tested $(30 \mu \mathrm{M})$. This is unsurprising, as N-terminal structural modifications of vancomycin often hinder the essential interactions with the D-Ala-DAla motif in the cell wall that are required for antimicrobial activity ${ }^{23,28}$. A loss of antimicrobial activity was also observed for the V-linked compound (V7), which increased with linker length (V8-9). This loss is possibly explained by the bulky attachment altering vancomycin dimerization that occurs through these sugar groups and that again is important for antimicrobial activity ${ }^{29,30}$. The C-linked conjugate with no spacer $(\mathbf{C 1})$ retained the highest antimicrobial activity of any of the antibiotic-chemoattractants, albeit only half the activity of vancomycin. Similar to the V-linked conjugates, there was a loss of antimicrobial activity with the inclusion of PEG spacers (C2-3).

This reduction in antimicrobial activity would normally suggest that these would not be an effective treatment strategy. However, the key outcome here was that the vancomycin core still bound to the cell wall of $S$. aureus independently of the antibiotic resistance state. Given the antimicrobial activity profiles of all conjugates, fPep attachment through C-linkage with no PEG linker was chosen for further analysis to maximize the potential for bacterial binding. The addition of chemical moieties to the C-terminus of vancomycin has previously been seen to be an effective strategy to enhance antimicrobial activity ${ }^{23,31-35}$. This decision also considered the current drug approval environment that favors the assessment of direct antimicrobial activity for such compound classes.

Determining the effect of fPep sequence on neutrophil recruitment. Having established vancomycin as a viable chassis for antibiotic-chemoattractant conjugates through C-terminal attachment, we next turned to optimization of the fPep component and investigated the effect of different fPep sequences on neutrophil recruitment. The sequence of fPeps are important for both potency and differential activation of the two main formyl peptide receptors (FPRs) of neutrophils, FPR1 and FPR213. Whilst high-resolution structures of FPRs are now available 36,37 most of what is known regarding the diversity of sequences recognized by specific FPRs has been defined by fPep libraries ${ }^{38-40}$. However, the influence of fPep sequence on downstream chemotaxis and phagocytosis remains largely unknown ${ }^{14,41}$. We therefore investigated the effect of altering fPep sequence on neutrophil recruitment.

The fMLFG (FP1) sequence is a potent chemoattractant, resulting in peak neutrophil chemotaxis at $100 \mathrm{nM}$. We postulated that our antibiotic-chemoattractants could require an fPep that would maximally activate neutrophils at different concentrations to FP1 in order to ensure optimal fPep-induced neutrophil recruitment from our conjugates without detrimental overstimulation $^{42,43}$. To identify the optimum fPep, we generated a peptide library based upon the fMLFG sequence using combinatorial peptide synthesis on SynPhase ${ }^{\mathrm{mm}}$ lanterns (Fig. 2a). Each lantern was tagged and common synthetic steps could be achieved following a split/mix strategy to speed the elaboration of the library. For library design, we modified each of the residues in the fMLFG sequence using a variety of proteinogenic and nonproteinogenic amino acids (Fig. 2 \& Supplementary Table 2) that covered a range of hydrophobicity and size profiles (Supplementary Fig. 2). Using a transwell assay we examined the recruitment of human neutrophils to these peptides (1-1000 nM) and observed that this library could be classified into five different profiles depending on concentration of peak recruitment (Fig. 2b-f). No obvious trends connected the chemotaxis profile observed to the position of the change, solvent-accessible surface area, or the hydrophobicity of the fPep. This was despite the wide range of hydrophobicity profiles tested (ranging from a $\log (\mathrm{P})$ of -2.5 to 2.5 compared to the original fMLFG sequence) and changes in the solvent-accessible surface area (Supplementary Fig. 3). Oxidation and hydrolysis are two common events that can render these sequences inactive. For this reason, we included norleucine as a replacement for methionine as it is resistant to oxidation, thus producing a longer-lived fPep that could benefit the conjugate ${ }^{44}$. Furthermore, peptides that did not contain the formyl group or had a glutamic acid instead of phenylalanine in the position three of the peptide did not lead to neutrophil recruitment at any concentration tested (Supplementary Fig. 4).

We next investigated if the attachment of these peptides onto vancomycin altered their ability to induce neutrophil chemotaxis. Following the strategy described above, we linked representative peptides displaying each of the five chemotactic profiles onto vancomycin (C-linked) and examined neutrophil recruitment in response to these compounds. The attachment of fPeps to vancomycin led to a general shift in neutrophil recruitment towards higher concentrations. This phenomenon was also observed with conjugation of the archetypal fMLFG sequence, likely due to the interaction of the bulky vancomycin molecule altering neutrophil FPR activation. Interestingly, these changes led to altered profiles for fPeps where the maximal chemotaxis was observed at either 100 or $1000 \mathrm{nM}$, irrespective of the initial chemotaxis profile for the free fPep. In contrast, fPeps that initially induced peak chemotaxis at 100 and $1000 \mathrm{nM}$, fMLFP (FP26) and fMLFK (FP21, Fig. 2e), displayed unchanged profiles once conjugated to vancomycin (C16-17). For the fMChaFG peptide (FP11), which displayed peak recruitment at 10 and $100 \mathrm{nM}$ (Fig. 2c), attachment to vancomycin abolished recruitment below $10 \mathrm{nM}$ (C11). Vancomycin alone or linked to MLFG (C12) led to no neutrophil recruitment at any of the concentrations tested (Supplementary Fig. 3).

The peptides fMLFL (FP19, Fig. 2d) and fMLYG (FP12, Fig. 2f), which recruited low numbers of neutrophils across the concentration range tested, also led to the same behavior when conjugated to vancomycin (C18-19). These sequences were chosen for conjugation, as the low levels of neutrophil recruitment made them ideal for testing whether they could improve bacterial killing whilst avoiding potentially deleterious overstimulation of the immune response ${ }^{42,43}$. These conjugates C18-19, along with $\mathrm{fMLFG}=\operatorname{van}(\mathbf{C 1})$ and $\mathrm{fMChaFG}=$ van (C11) were all found to have MIC values of $1.8 \mu \mathrm{M}$ against both MRSA and MSSA strains tested. This indicates that the sequence of the formylated peptide attached to vancomycin does not alter 
a

fPep screen

$\mathrm{fPep}=$ van screen

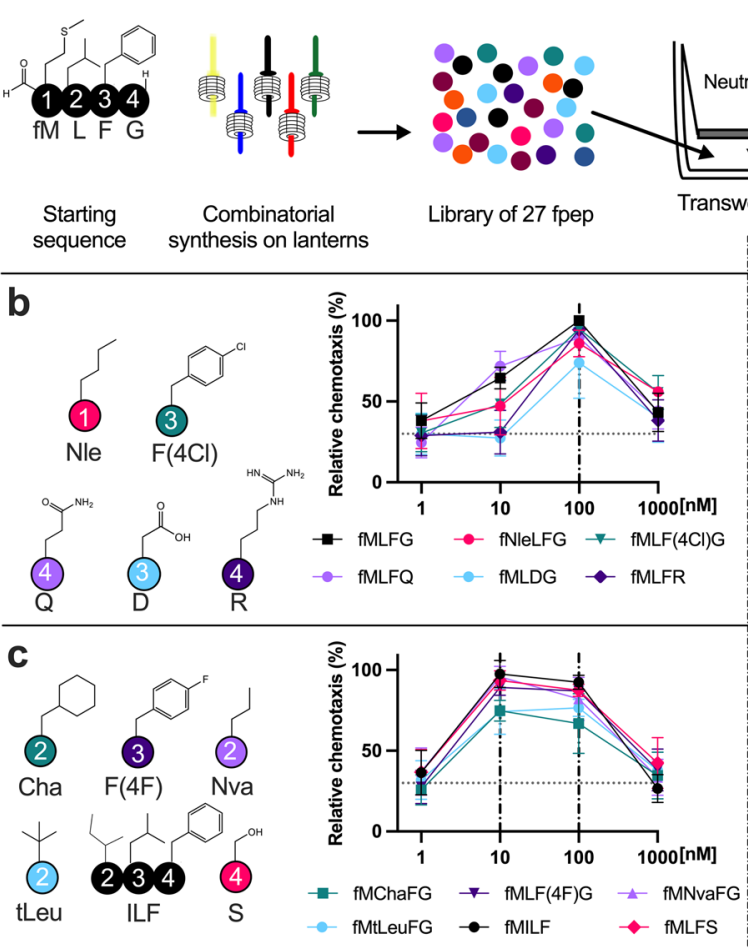

d
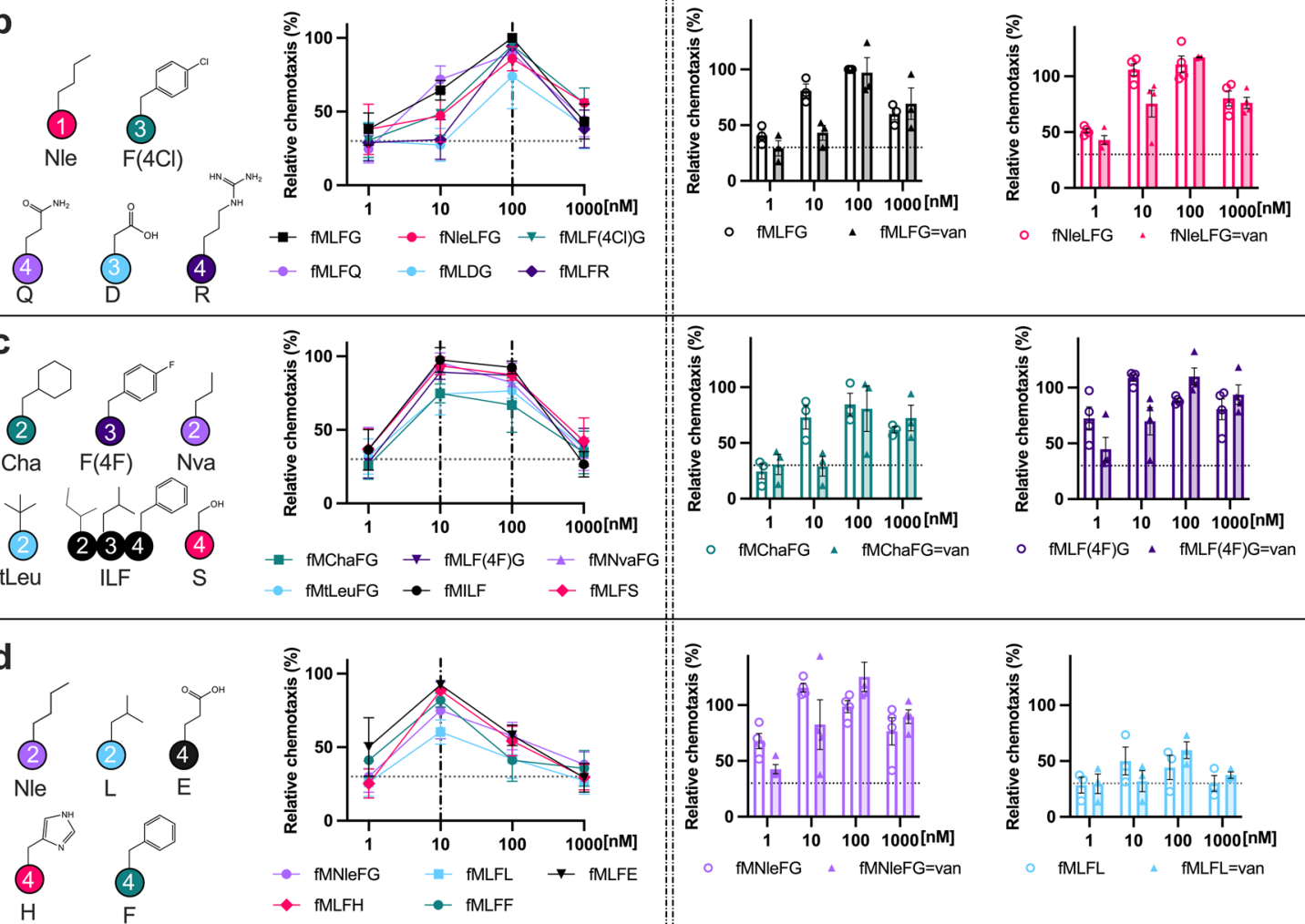

$\circ \quad f M L F$
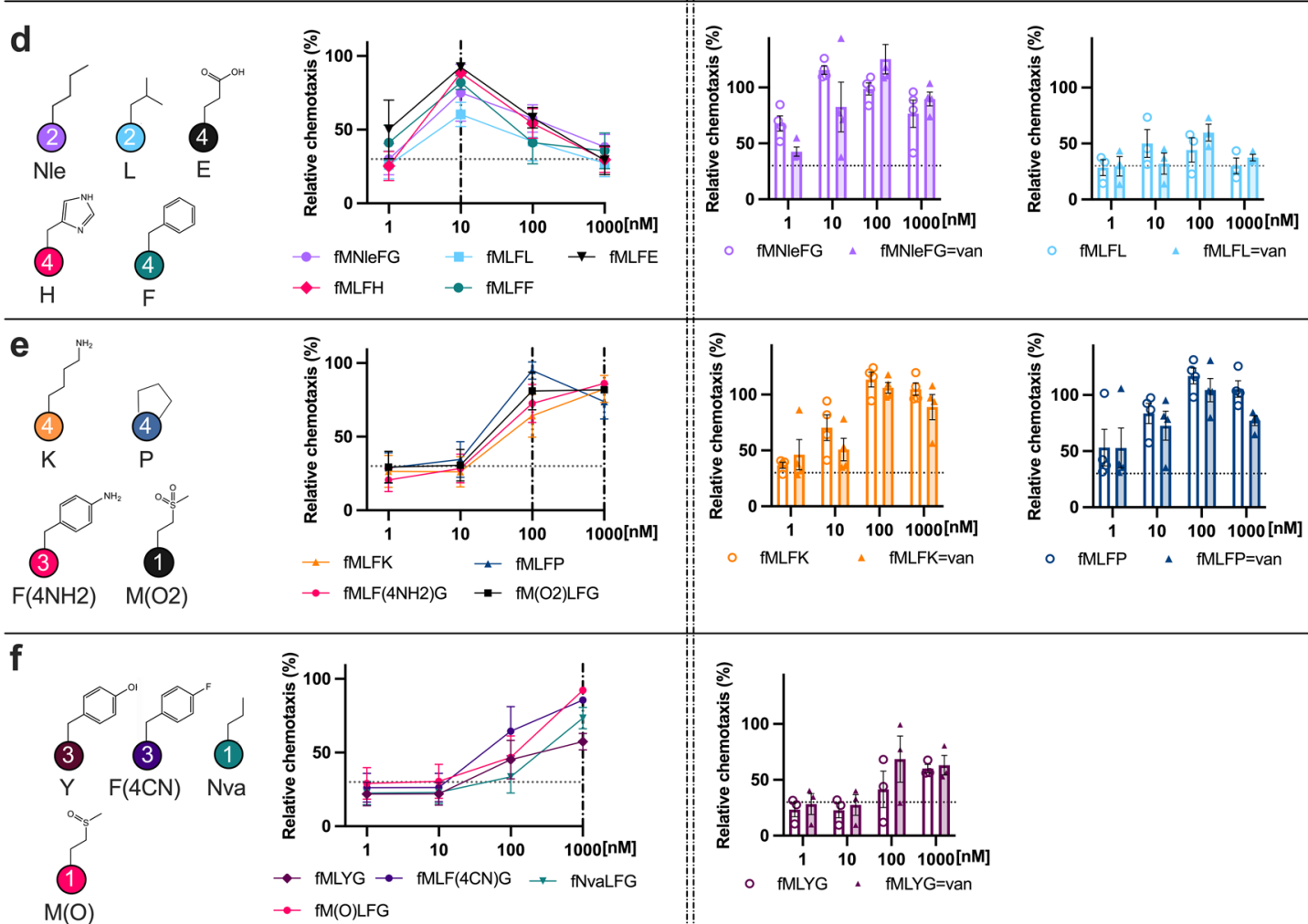

antimicrobial activity, unlike that seen with varying the attachment site of the fPep to vancomycin. It also allows the direct comparison of the neutrophil killing activity of these different conjugates as they possess the same level of direct antimicrobial activity against the bacterial strains tested.

Testing neutrophil recruitment and direct antimicrobial killing separately allowed us to independently optimize the individual

activities of our antibiotic chemoattractant. Next, we sought to explore how direct antibiotic activity and binding, combined with induction of neutrophil recruitment, might lead to enhanced killing of $S$. aureus.

Effects of fPep sequence on neutrophil recruitment and phagocytosis of $S$. aureus. Using an infection-on-a-chip 
Fig. 2 The fPep sequence either free or linked to vancomycin affects neutrophil recruitment. a Schematic depiction of the process to synthesize and test a library of fPeps centered on the fMLFG sequence. The initial library of $27 \mathrm{fPep}$ were generated by combinatorial solid-phase peptide synthesis on SynPhase ${ }^{\mathrm{TM}}$ lanterns. A transwell assay was used to determine the chemotaxis of human neutrophils to these peptides in a concentration range of 1-1000 nM. The library of fPeps were grouped into 5 profiles (line graphs, B-F, $n=3$ biological independent experiments, error bars are SEM) based on the concentration of the fPep that resulted in the greatest recruitment of neutrophils. Peak recruitment was observed at: $100 \mathrm{nM}$ (b), both 10 and $100 \mathrm{nM}$ $(\mathbf{c}), 10 \mathrm{nM}(\mathbf{d}), 100$ and $1000 \mathrm{nM}(\mathbf{e})$, and $1000 \mathrm{nM}(\mathbf{f})$. The changes in $\mathrm{fMLFG}$ sequence are indicated by the altered residue number $(1=\mathrm{fM}, 2=\mathrm{L}, 3=\mathrm{F}$, $4=\mathrm{G}$ ), with the side chain of the switched amino acid indicated. Representative fPeps from each of these profiles were linked to the $C$-terminus of vancomycin via click chemistry and retested for the ability to recruit neutrophils using transwell assay (bar graphs, solid bars represent conjugate). Chemotaxis was calculated relative to the no protein control and $100 \%$ chemotaxis set as the neutrophil recruitment observed to $\mathrm{fMLFG}$ at $100 \mathrm{nM}$ for each donor, $n=3-4$ biological independent experiments, error bars are SEM. Dotted line on graphs indicates average recruitment to $0 \mathrm{nM}$.

microfluidic device, we next quantified neutrophil recruitment, phagocytosis of bacteria through pHrodo fluorescence and growth of GFP expressing $S$. aureus in response to our antibioticchemoattractant (Fig. 3a) ${ }^{45}$. Curiously, the kinetics of neutrophil recruitment were slightly faster for the conjugated fMLFG compound (C1) compared to the free peptide (FP1, Fig. 4), while fMChaFG showed similar kinetic profiles for both the free (FP11) and conjugated (C11) peptides (Fig. 3). Neutrophil chemotaxis into the microchambers occurred from the start of the assay, while pHrodo fluorescence, indicating the phagocytosis of bacteria, was detected after $60 \mathrm{~min}$ (Fig. 3). Importantly, the increased recruitment of neutrophils into the chamber mediated by the antibioticchemoattractants resulted in enhanced control of $S$. aureus growth compared to vancomycin or fPep alone (Fig. 3 and Supplementary Fig. 5). The control of $S$. aureus growth here is due to the neutrophil action, rather than the direct antimicrobial activity of the compounds: this was confirmed by the observations that the MLFG= van compound, which does not result in neutrophil recruitment, but that displays similar direct antimicrobial activity as $\mathbf{C 1}$ and $\mathbf{C 1 1}$, resulted in no observable growth inhibition of $S$. aureus by neutrophils in the microfluidic assay(Supplementary Fig. 5).

Although there was more rapid recruitment of neutrophils observed for the fMLFG antibiotic-chemoattractant (C1), there was only a slight increase in phagocytosis over the free fPep (FP1). In contrast, fMChaFG (FP11) and its corresponding antibiotic-chemoattractant (C11) generated similar neutrophil recruitment into the microchamber. However, the antibioticchemoattractant induced more rapid phagocytosis and higher levels of total phagocytosis compared to the free peptide (Fig. 3e). This indicates that the fMChaFG antibiotic-chemoattractant (C11) increased the number of bacteria phagocytosed per neutrophil (observed with all neutrophil donors to different extents), which was not observed for the fMLFG conjugate (Fig. 3f). For antibiotic-chemoattractants based on fMLFL (C18) and fMLYG (C19), enhancement of phagocytosis appeared to be donor specific, although quantification of this effect was limited by their lower levels of neutrophil recruitment (Fig. 3f).

To directly demonstrate that antibiotic-chemoattractants bind to $S$. aureus and result in an fPep gradient that stimulates neutrophil recruitment, we next incubated the fMLFG antibioticchemoattractant (C1) or the free fPep (fMLFG, FP1) with $S$. aureus bioparticles, before washing the samples to the extent where no neutrophil recruitment was observed for the free peptide treated sample (fPep estimated to be less than $0.1 \mathrm{nM}$ ). Strikingly, the washed antibiotic-chemoattractant treated $S$. aureus bioparticles induced recruitment of neutrophils to the same extent as $100 \mathrm{nM}$ fPep (Fig. 3g). This demonstrates both initial binding and secondary chemoattractant gradient formation following treatment with these antibiotic-chemoattractants.

Antibiotic-chemoattractants interact with FPR1 and FPR2. The sequence of fPeps are known to be important for both potency and differential activation of the two main FPRs present on neutrophils, FPR1 and FPR2. The fPeps interact with two FPRs expressed on human neutrophils, and although these G-protein coupled receptors share $69 \%$ sequence identity, they have distinct preferences for fPep binding. In general, FPR1 recognizes short fPeps with high affinity. In contrast, FPR2 is more of a promiscuous receptor that recognizes a diversity of ligands, including longer fPeps within its larger binding pocket ${ }^{36,37}$. Here we have observed that there is a sequence-specific enhancement phagocytosis by $\mathrm{AMChaFG}$ conjugate (C11), a downstream result of FPR activation. Such enhanced phagocytosis could well be due to the larger, more hydrophobic sequence selectively activating FPR2, which has a larger binding pocket and accepts more hydrophobic peptides than the FPR $1^{46}$. To examine this, we added the Rhodamine B labelled FPR2 antagonist, PBP10 (RhB-PBP10), to human neutrophils to determine which fPeps and conjugates could compete with this FPR2 antagonist (Fig. 4a). Conjugation of fMLFG (C1) resulted in a decrease in RhBPB10 binding compared to the free fMLFG peptide (FP1), suggesting that conjugation resulted in the AMLFG sequence becoming a stronger FPR2 binder, showing similar levels of competition to the known FPR2 preferential binder fMIVIL. This is in agreement with previous observations where other fPep conjugates that changed binding preference from FPR1 to FPR2 due to the increase in the size of the peptide 47,48 . The free $\mathrm{fMChaFG}$ peptide was able to compete with RhB-PB10 binding, while in contrast the conjugation of the $\mathrm{AMChaFG}$ peptide resulted in an inability to compete with RhB-PB10. Here, treatment with the conjugate form (C11) was shown to lead to similar levels of RhB-PBP10 binding as the known preferential FPR1 binder AMLF, the negative control.

Binding to FPRs can lead to the activation of a range of downstream pathways. We examined two of these pathways through monitoring the phosphorylation of ERK, and the mobilization of calcium. Using CHO cells overexpressing either human FPR1 or FPR2, the levels of ERK phosphorylation and the calcium mobilization were monitored in response to both PMLFG and $\mathrm{fMChaFG}$ in both the free and conjugated forms (FP1, C1 and FP11, C11), as has been previously reported ${ }^{49,50}$. All peptides resulted in a peak-response time of 5 min for ERK phosphorylation (Fig. 4c). Whilst this activation quickly dissipated in the FPR1 cell line, it appears to be relatively more sustained in the cells expressing FPR2 compared to the fMLF control. $\beta$-arrestins regulate GPCR signaling through receptor desensitization and internalization $^{51}$, and therefore this sustained FPR2 signaling through ERK may be due to decrease in $\beta$-arrestin dependent MAPK kinase (ERK1/2) activation in this pathway compared to the fMLF. The concentration-response curve was also examined at this peak response of $5 \mathrm{~min}$. In general, conjugation of both fPeps resulted in a lower potency for FPR1 activation compared to their free counterparts for both ERK phosphorylation (10-fold increase, Fig. $4 \mathrm{~d}$ ) and calcium mobilization ( $>90$-fold decrease in $\mathrm{pEC}_{50}$, Fig. $4 \mathrm{~b}, \mathrm{~d}$ ) and an increase in potency for FPR2 calcium mobilization ( $>90$-folds increase in $\mathrm{pEC}_{50}$, Fig. $4 \mathrm{~b}, \mathrm{e}$ ). Despite lacking the ability to outcompete RhB-PB10 in binding to FPR2, 

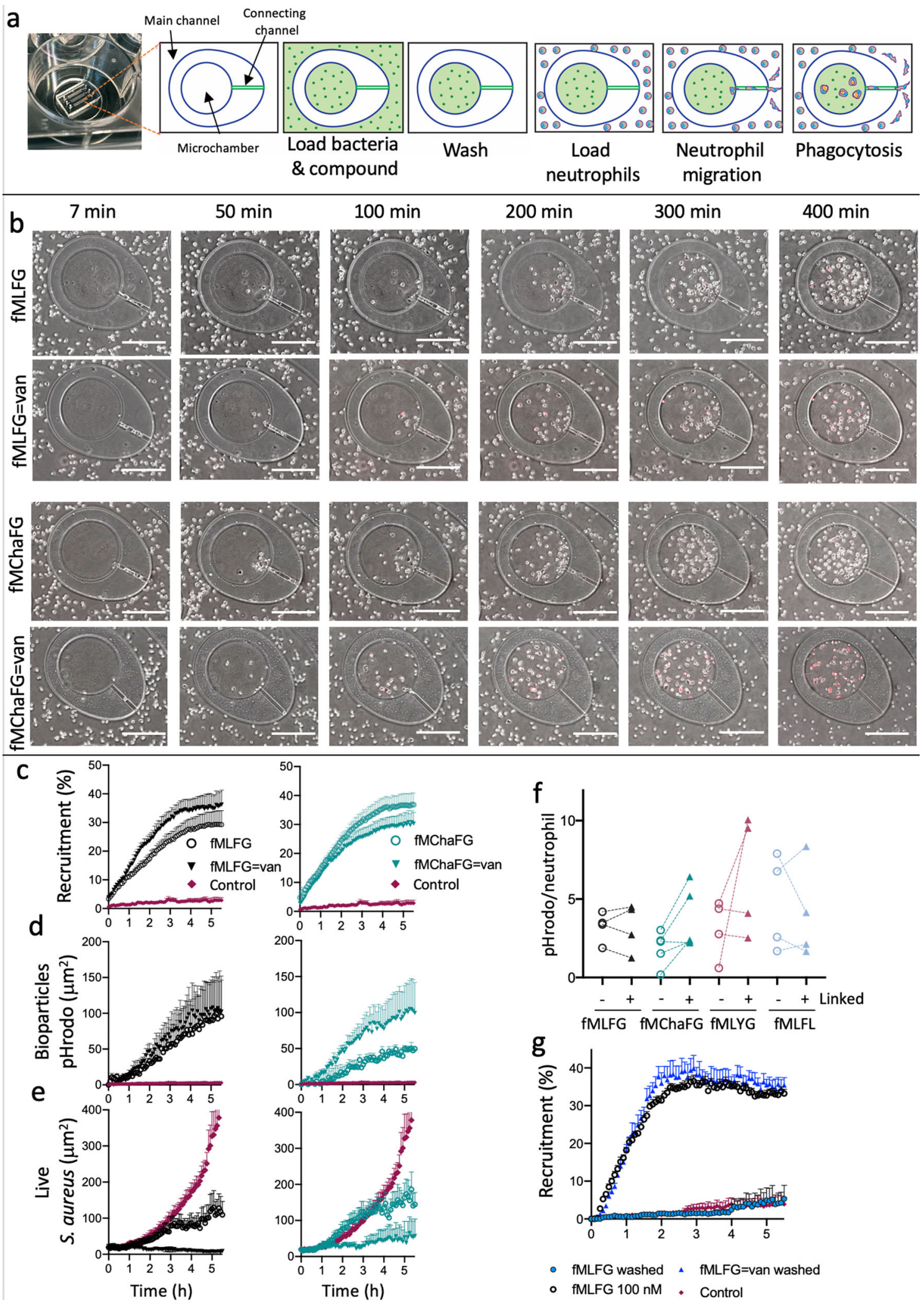

the fMChaFG conjugate (C11) demonstrated ERK1/2 phosphorylation and calcium mobilization in FPR2 expressing cells similar to the fMLFG conjugate (C1).

Antibiotic-chemoattractants reduce inflammation and bacterial load in a mouse model of S. aureus pneumonia. Based on the infection-on-a-chip results, our most promising antibioticchemoattractant (fMChaFG = van, C11) was tested for efficacy in a MRSA mouse pneumonia model (Fig. 5a). Prior to these experiments, the conjugate $\mathbf{C 1 1}$ was confirmed to recruit mouse neutrophils using the transwell-based assay; this was tested as FPR sequence specificity differs between mouse and human. The mouse pneumonia model was performed by first establishing an 
Fig. 3 Linking a fPep to vancomycin enhances the phagocytosis activity of neutrophils. a The infection-on-a-chip device used to monitor neutrophil migration and phagocytosis over time present in a six-well plate. Within this microfluidic device the egg-like microchambers in the main channel were loaded with S. aureus plus compound, washed, and neutrophils loaded. A chemotactic gradient of compound establishes from the microchamber along a single cell wide connecting channel. Bacteria are labelled with the $\mathrm{pH}$ activated dye, pHrodo to allow monitoring of phagocytosis. $\mathbf{b}$ Time course of neutrophils migrating into the microchamber containing S. aureus bioparticles labelled with pHrodo, in the presence of free fPep (FP1, or FP11) or conjugated (C1 or C11) at 1000 or $0 \mathrm{nM}$ (control). Images are representative of four donors; scale bars $10 \mu \mathrm{m}$. Quantification of neutrophils having migrated into the microchamber (c) along with the area of pHrodo fluorescence (d), or the area of GFP fluorescence as a measure of S. aureus growth (e) determined in the presence of conjugated (triangles, C1 or C11) or free fPep (circles, fMLFG FP1, black; and fMChaFG FP11, teal; at 1000 or 0 nM, control). Data are the average of four donors, with error bars being SEM. $\mathbf{f}$ The sequence of the conjugate effects efficiency of neutrophil phagocytosis as determined by the area of pHrodo fluorescence per neutrophil recruited into the microchamber at $2 \mathrm{~h}$. Data from each donor is linked by dotted lines. g fMLFG conjugated to vancomycin ( $\mathrm{fMLFG}=$ van C1) or free fPep (FP1) were incubated with S. aureus bioparticles and washed before loading into the microchamber. The washed conjugate treated S. aureus recruited similar levels of neutrophils compared to samples with $100 \mathrm{nM}$ fMLFG (open circles). Data are representative of three donors, error bars are SEM.

MRSA infection in the lung, prior to treatment being administered $1 \mathrm{~h}$ postinfection intranasally with a dose of $0.2 \mathrm{mg} / \mathrm{mouse}$ equivalent of vancomycin. In regard to the area under the concentration-time curve (AUC) of total vancomycin in serum, this dose is 5 -fold less than the standard $100 \mathrm{mg} / \mathrm{kg}$ equivalent of the clinical dose of vancomycin in humans.

Promisingly, treatment with this low dose of antibioticchemoattractant resulted in a 2 -fold reduction in MRSA load in the lungs compared to vancomycin alone at 12 hpi (Fig. 5b). Unsurprisingly, histopathology revealed that the lung tissue of the vehicle control mice had the most severe and widespread inflammation and bacterial load, which resulted in dramatic loss of alveoli structure (Fig. 5c, d). In these mice, inflammation was centered on the alveoli, where large numbers of neutrophils, alveolar macrophages, and both extracellular and intracellular bacteria were found. Mice in the vancomycin control treatment group showed reduced severity of inflammation and lower bacterial load, although lung damage was still observed. The fPep and antibiotic-chemoattractant treatment groups both showed reduced inflammation and bacterial numbers compared to vancomycin treatment, as the inflammation did not extend into the lumen of the bronchioles and bronchi (Fig. 5e). Importantly, the antibiotic-chemoattractant treatment alone exhibited a combination of reduced bacterial load and improved tissue pathology.

\section{Discussion}

In this work, we successfully explored the concept of antibioticchemoattractants to enhance the neutrophil response against the deadly immune-evading pathogen $S$. aureus. In choosing vancomycin as the targeting moiety in these constructs, we were able to take advantage of its cell wall binding properties to establish a gradient. Furthermore, the clinical use and development of glycopeptide antibiotics such as vancomycin provides avenues for improving the properties of these agents and also offers the potential for scaled production in the future ${ }^{19}$. As a chemotactic agent, the use of fPeps provided the opportunity to explore a wide compound space in a facile manner using the solid-phase synthesis of peptide libraries, which showed that modified sequences display different kinetics of neutrophil recruitment and phagocytosis for different antibiotic-chemoattractants. This offers the possibility to tune these responses as a function of the fPep payload sequence, with the most promising conjugates fMChaFG (C11) not only stimulating effective chemotaxis but also increasing phagocytosis per neutrophil for all donors.

FPR are promiscuous receptors that bind to a range of ligands that are structurally and functionally distinct. Biased agonism or ligand-dependent agonism is an emerging field of GPCR research, which has the potential for the development of drugs with selective beneficial effects. We have previously demonstrated that biased agonism is an important molecular pharmacology consideration for these receptors ${ }^{49,52}$, with biased agonists for FPRs known ${ }^{49}$. This provides the potential for designer FPR ligands to bias aspects of FPR activation. Understanding how altered fPep sequences lead to differential FPR binding and intracellular signaling is highly important in understanding their effects on the host defense, potentially as means to decode "danger sensing". Activation of these FPRs leads to not just neutrophil chemotaxis but also to release of various compound (reactive oxygen species, host defense peptides, chemokines), formation of neutrophil extracellular traps ${ }^{53}$ and enhance phagocytosis of $S$. aureus ${ }^{14,41}$. How FPRs (in particular FPR2) transmit signals from their diverse ligands to the intracellular space to play distinct, sometimes opposite, physiological roles in inflammation is largely unknown. Here, we observed that whilst the widely investigated fPep sequence fMLFG led to FPR1 activation as anticipated, substitution of the second Leu residue with Cha (fMChaFG) was sufficient to alter the activation profile of this peptide to favor FPR2 activation. However, upon conjugation to vancomycin, the fMChaFG conjugate was unable to compete effectively with the known FPR2 agonist, RhB-PB10. These results suggest a complex relationship between receptor binding and downstream activation events, which require further investigation to determine how the fMChaFG conjugate affords enhanced phagocytosis. This process will involve untangling the tangled web of ligand recognition, biased signaling, allosteric modulation, receptor cross-talk, as well as hetero and homo receptor dimerization in these important G-coupled protein receptors ${ }^{54}$.

We also noted differences between the human donors in terms of neutrophil chemotaxis and phagocytic responses to the different fPep sequences (in particular fMLFL and fMLYG). These different donor responses could be explained by the variation in FPR number and function that are present in the human population due to both polymorphisms within the FPR1 gene and the three isoforms of FPR $1^{46}$. These genetic variations lead to differences in response to fPep sequences ${ }^{55,56}$ and in turn the FPR encoded has therefore been linked to the response of individuals to infections ${ }^{57-59}$. The modular nature of the antibioticchemoattractants provides a potential pathway toward personalized treatment of an infection that takes into account the FPR genotype to select the optimum fPep sequence for the patient. This complex chain of effects also highlight the importance of using the correct tools to validate the multifaceted activities of these immunotherapeutics, which are otherwise lost in approaches that utilize "averaged" donor responses.

The infection-on-a-chip data allowed us to identify the optimal antibiotic-chemoattractant to test in a mouse model of MRSA pneumonia. This revealed that antibiotic-chemoattractants can function in the whole host environment, and at a five-fold lower concentration compared to standard vancomycin treatment for 
a

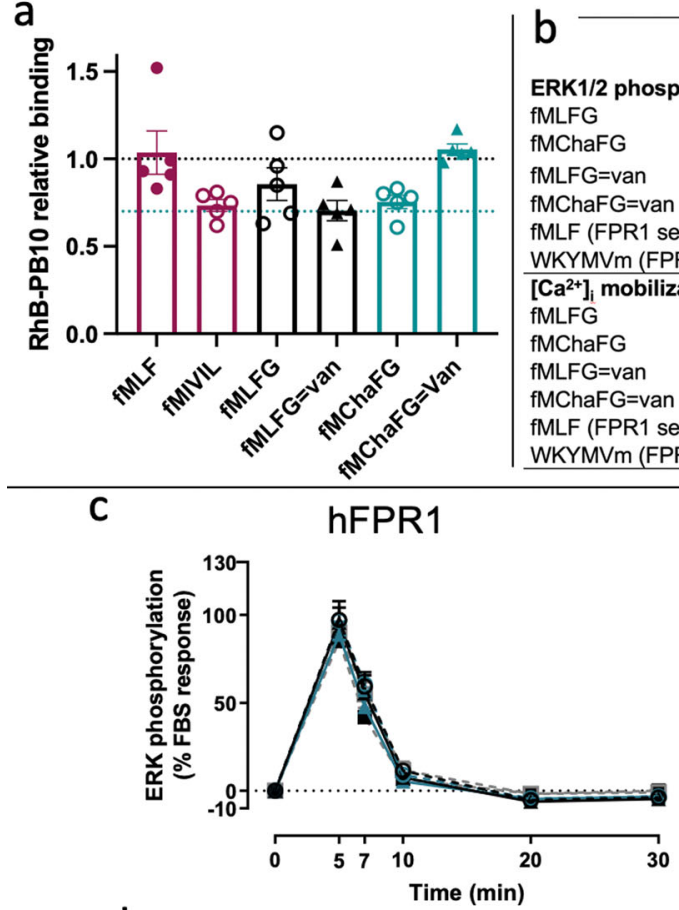

d
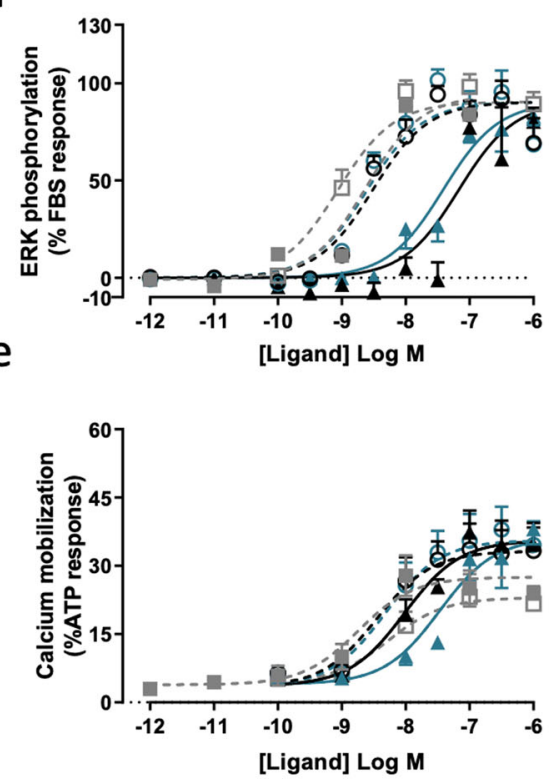

\begin{tabular}{|c|c|c|c|c|c|}
\hline \multicolumn{2}{|c|}{ FPR1 } & \multicolumn{4}{|c|}{ FPR2 } \\
\hline $\mathrm{pEC}_{50}$ & $E_{\max }(\%)$ & $\mathbf{n}$ & $\mathrm{pEC}_{50}$ & $E_{\max }(\%)$ & $\mathbf{n}$ \\
\hline $8.60 \pm 0.09$ & $86 \pm 8$ & 5 & $5.54 \pm 0.38$ & $82 \pm 7$ & 5 \\
\hline $8.66 \pm 0.07$ & $90 \pm 8$ & 5 & $5.73 \pm 1.05$ & $81 \pm 9$ & 5 \\
\hline $7.41 \pm 0.15^{*}$ & $77 \pm 8$ & 4 & $6.20 \pm 0.08$ & $99 \pm 9$ & 4 \\
\hline $7.50 \pm 0.20^{\#}$ & $83 \pm 3$ & 5 & $6.91 \pm 0.72$ & $84 \pm 4$ & 5 \\
\hline $9.04 \pm 0.12$ & $98 \pm 11$ & 3 & $<4$ & $99 \pm 27$ & 3 \\
\hline $8.68 \pm 0.14$ & $98 \pm 9$ & 3 & $9.34 \pm 0.10$ & $110 \pm 7$ & 3 \\
\hline $8.17 \pm 0.29$ & $27 \pm 4$ & 6 & $5.87 \pm 0.26$ & $34 \pm 7$ & 6 \\
\hline $8.02 \pm 0.21$ & $27 \pm 4$ & 6 & $5.88 \pm 0.21$ & $43 \pm 13$ & 6 \\
\hline $7.62 \pm 0.36^{* *}$ & $34 \pm 8$ & 7 & $6.91 \pm 0.24^{\star * *}$ & $45 \pm 10$ & 7 \\
\hline $7.43 \pm 0.11^{\# \#}$ & $24 \pm 3$ & 7 & $7.24 \pm 0.07$ & $27 \pm 3$ & 7 \\
\hline $8.11 \pm 0.12$ & $18 \pm 3$ & 3 & $6.18 \pm 1.29$ & $18 \pm 4$ & 3 \\
\hline $8.48 \pm 0.61$ & $24 \pm 4$ & 3 & $9.20 \pm 0.15$ & $31 \pm 5$ & 3 \\
\hline
\end{tabular}

hFPR2
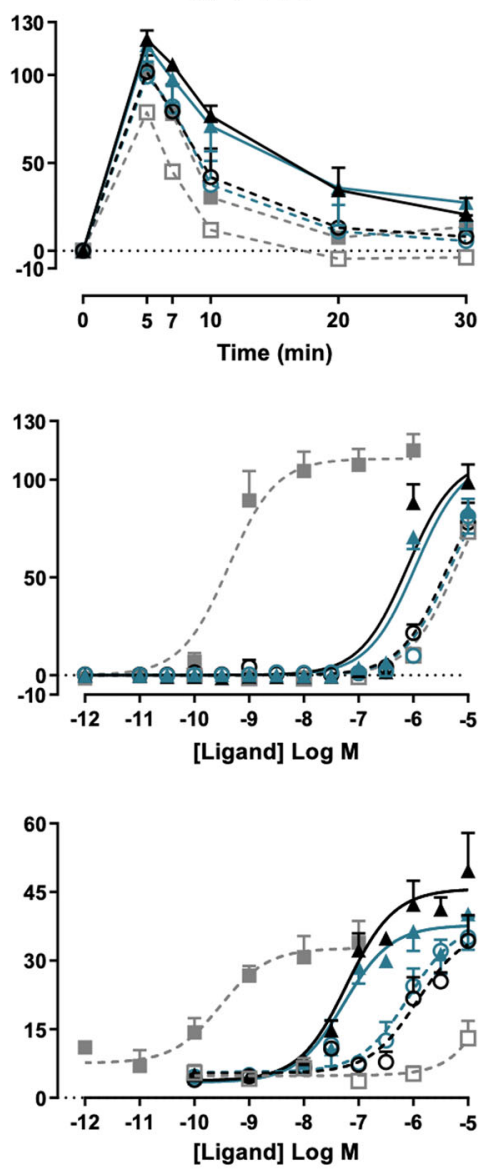

๑. fMLFG
$\cdot$ fMChaFG $-\boxminus \cdot$ PMLP
- WKYMVm

Fig. 4 Interaction of fPeps with human FPRs and activation of downstream pathways. a Competition binding to human neutrophils between the FPR2 antagonist RhB-PB10 and different fPeps. fMLF binds preferentially to FPR1 and was used as a negative control, while fMIVIL binds preferentially to FPR2. The peptides fMLFG and FMChaFG and their corresponding conjugates were examined for their ability to compete with RhB-PB10 binding to neutrophils, $n=5$ biologically independent experiments, with error bars of SEM. $\mathbf{b}$ CHO cells overexpressing human FPR1 or 2 were assessed for the downstream activation of either ERK1/2 phosphorylation or $\mathrm{Ca}^{2+}$ mobilization $\left(\left[\mathrm{Ca}^{2+}\right]_{i}\right)$ with $\mathrm{E}_{\max }$ and $\mathrm{pEC} \mathrm{C}_{50}$ calculated. Data presented as mean $\pm \mathrm{SEM}$ and an unpaired $t$ test were used to compare the effects of the fMLFG and fMChaFG in free and conjugated forms. $\mathrm{n}$ indicates the number of biological replicates with twotailed $p$-values indicated by ${ }^{\star} p=0.0002,{ }^{\star \star} p=0.02,{ }^{\star \star \star} p=0.01$ vs. $f M L F G,{ }^{\#} p=0.0006,{ }^{\# \#} p=0.01, \# \# p=0.000048$ vs fMChaFG. c Time course of ERK1/2 phosphorylation $(n=4$ biological replicates performed in triplicate, error bars are SEM). $\mathbf{d}$ Concentration-response curves for $p E R K 1 / 2$ were determined at $5 \mathrm{~min}$, the peak-response time point determine during the time course. The positive controls were the hexapeptide Trp-Lys-Tyr-Met-Val-DMet (WKYMVm), a specific FPR2 agonist, along with N-formyl-Met-Leu-Phe (fMLF) as a specific FPR1 agonist. Data were normalized to vehicle and the response elicited by 10\% FBS, with nonlinear regression curve plotted with error bars of SEM, $(n=3-5$ experiments, each performed in duplicate). e Intracellular calcium mobilization was determined in response to fPeps in $\mathrm{CHO}$ cells overexpressing either human FPR1 or FPR2. The fluorescence signal was normalized to the cellular response mediated by the positive control, adenosine triphosphate (ATP) at $100 \mu \mathrm{M}$, plotted with nonlinear regression curve, with error bars of SEM, ( $n=3-7$ experiments, each performed in triplicate). 
a

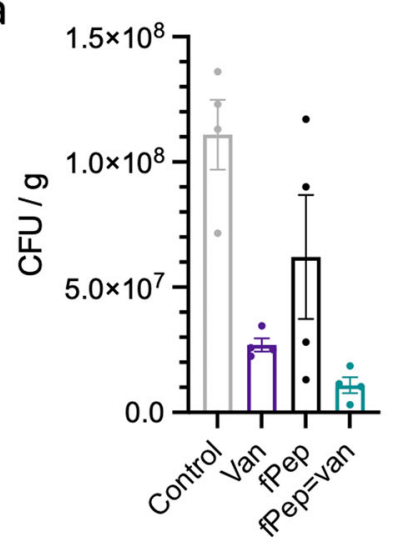

b

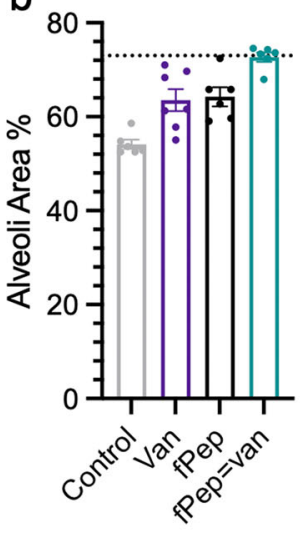

C

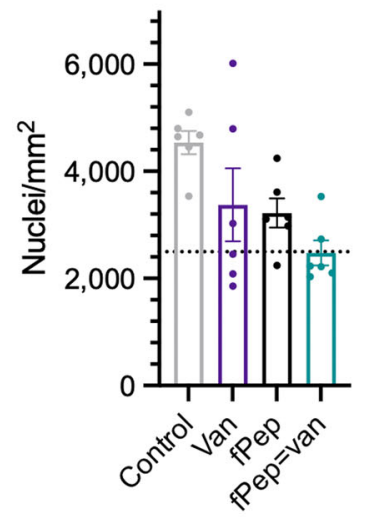

d

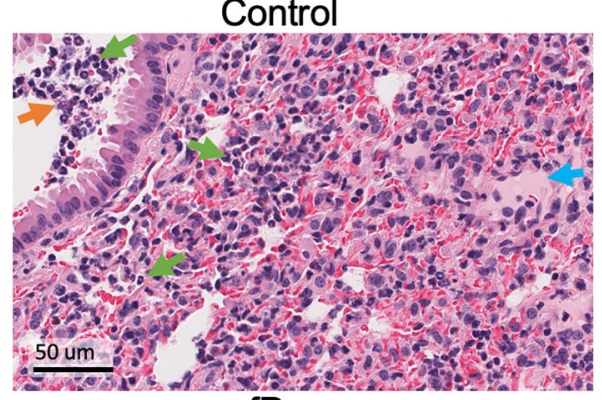

fPep

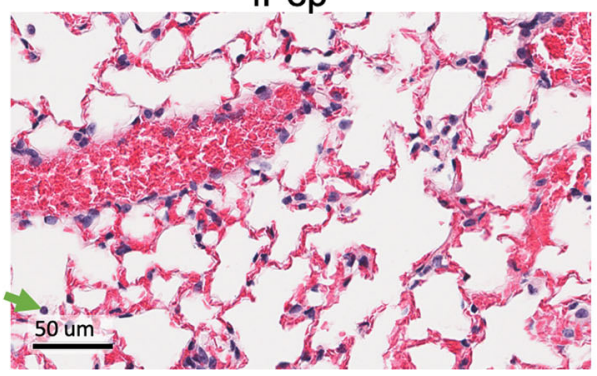

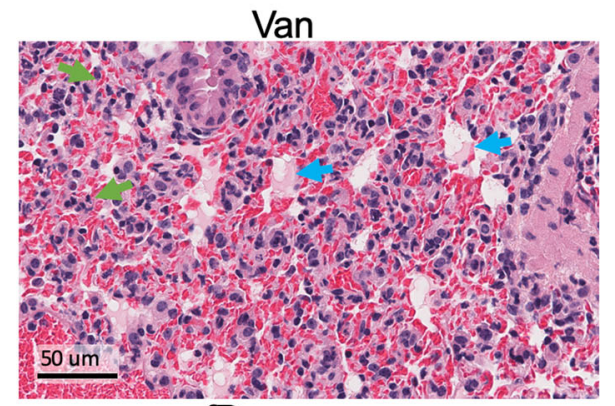

$\mathrm{fPep}=\mathrm{van}$

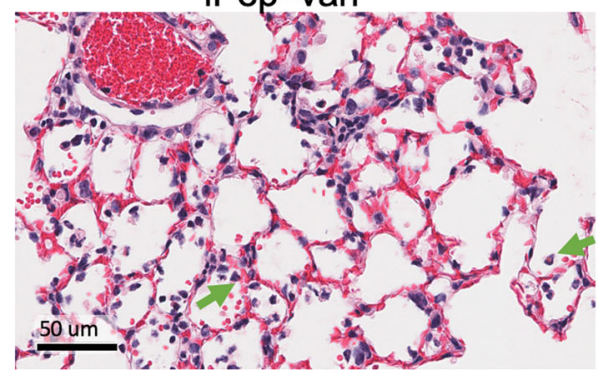

Fig. 5 fPep-vancomycin conjugate C11 reduces bacterial load and inflammation in a mouse pneumonia model. Eight-week-old female mice were infected by intranasal inhalation of $10^{7} \mathrm{CFU} \mathrm{S}$. aureus to induce pneumonia. One-hour postinfection (hpi) mice were given intranasal therapy at $0.2 \mathrm{mg} / \mathrm{mouse}$ equivalent of vancomycin or vehicle control (control). The lungs were collected at $12 \mathrm{hpi}$, and the bacterial load in the lung tissue of four mice per treatment group was determined (a). The fMChaFG conjugate ( $\mathbf{C 1 1}, \mathrm{fPep}=$ van) resulted in half the bacterial load compared to vancomycin (van) alone $(n=4$ mice/ group, error bars are SEM). The percentage of alveoli space (b) or the number of nuclei (c) was determined for hematoxylin and eosin-stained histology lung samples (d) from 6 fields of view of the outer lung lobes. Uninfected mouse lung is indicated by dotted line, error bars are SEM. Green arrows indicate neutrophils, blue arrows proteinaceous fluid, and orange arrows indicate cocci bacteria.

the clearance of MRSA pneumonia infections. This efficacy likely stems from the presence of a complete cohort of immune cells expressing FPR in the whole animal, which would contribute to the twofold enhanced clearance of the infection we observed in mice lungs. While we have focused on neutrophils in this study, FPR-expressing cells also include other important innate immune cells - macrophages, monocytes, and dendritic cells ${ }^{46}$, along with vascular cells ${ }^{60}$. It is important to note that in mice, fPep sequence specificity is different despite mice and human sharing high sequence homology between FPRs ${ }^{61}$. However, in combination with the infection-on-a-chip approach, these data provide strong support for the efficacy of our antibiotic-chemoattractants as a potential new therapy to overcome resistant bacterial infections.

Taken together, the fusion of an antibiotic targeting element with immune stimulation can provide a viable immunotherapeutic strategy to treat resistant $S$. aureus infections. Given the recognized problems with our reliance on natural antibiotics for infection control, it is vital that we explore new strategies to be able to control these resistant and highly virulent bacterial strains that threaten the cornerstones of modern medicine. Thus, the development of antibiotic-chemoattractants is not only important as an example of a potential strategy to control S. aureus infections but also provides a template for the experimental pipeline needed to identify and investigate these complex agents. The future of this approach lies in understanding the differences seen in host responses to these compounds and further exploring the ability of broadly effective compounds-such as the conjugates containing $\mathrm{fMChaFG}-$ to resolve different types of infections. Additionally, unlocking the specificity that results in not just chemotaxis, but also enhances neutrophil killing will be vital to ensure the success of such immunotherapeutics. The antibioticchemoattractants developed in this work demonstrate the potential to rejuvenate current antibiotics, and also to deliver a more nuanced approach to infection control by application of a suite of tools to assess the complex activity of these compounds. 


\section{Methods}

Vancomycin functionalization. The C-terminal carboxylic acid of vancomycin $\mathrm{HCl}(1 \mathrm{eq})$ was activated with COMU (1 eq) and triethylamine (TEA, $1 \mathrm{eq})$ in dimethylformamide (DMF) at $\sim 30 \mathrm{mM}^{27}$. After 5 min of pre-activation, either 3 -azidopronamine ( $2 \mathrm{eq})$ or propargylamine $(2 \mathrm{eq})$ were added. The reaction was stirred overnight, analyzed by LCMS (see below for conditions) after which the product was precipitated in diethyl ether and washed twice with diethyl ether, giving crude product in $\sim 80 \%$ yield that was purified by preparative RP-HPLC (see below for conditions). 5-azidopentanoic acid (1.1 eq) was pre-activated with COMU (1.1 eq) and TEA ( 2 eq) in DMF for $5 \mathrm{~min}$. This was added to a solution of vancomycin (HCL salt, 1 eq) dissolved in DMF at $~ 20-50 \mathrm{mM}$, stirred overnight and subsequently analyzed by LCMS. The product was precipitated in diethyl ether $\left(\mathrm{Et}_{2} \mathrm{O}\right)$ and washed twice with $\mathrm{Et}_{2} \mathrm{O}$, giving crude product in $\sim 85-98 \%$ yield. $\mathrm{LC}$ analysis allowed us to determine that the ratio between four compounds (Supplementary Fig. 5), namely vancomycin, azidopentanoyl loaded on the N-terminal, azidopentanoyl loaded on the vancosamine moiety and the compound resulting from double addition $(30 \%, 40 \%, 10 \%, 20 \%)$ that were all isolated by preparative RP-HPLC. N-terminal and vancosamine conjugation reactions were performed in one pot without protection; the resultant regioisomers were separated using RPHPLC, with the identity of each compound confirmed by small-scale hydrolysis The resultant mass difference caused by the loss of either vancosamine or the modified vancosamine thus allowed the identity of each compound to be confirmed.

Solid-phase peptide synthesis on SynPhase ${ }^{\mathrm{TM}}$ lanterns and rink-Amide resins. Peptides were synthesized on SynPhase ${ }^{\mathrm{mat}}$ PSDRAM lanterns and Rink-amide resin using an Fmoc strategy ${ }^{62}$. Deprotection steps were carried out in $20 \%$ piperidine/DMF for $20 \mathrm{~min}$, followed by four DMF washes (3 min for lanterns and $30 \mathrm{~s}$ for resins). Coupling was carried out with 4 eq. amino acid, $3.8 \mathrm{eq}$. HCTU, and 8 eq. DIEA for $1 \mathrm{~h}$ with shaking, followed by four DMF washes as described above. The N-terminal formylation was carried out after the final deprotection step by coupling formic acid ( $3 \mathrm{eq})$, with pre-activation achieved in the presence of EDC. $\mathrm{HCl}(3 \mathrm{eq})$ and HOBt $(3 \mathrm{eq})$ in DCM for $15 \mathrm{~min}$ at $4{ }^{\circ} \mathrm{C}$. Afterwards, DIEA (1 eq) was added to maintain a basic $\mathrm{pH}$ for the coupling to proceed and the reaction left overnight at room temperature. For the formylated peptide library, a split/mix method was performed where lanterns were mixed for common steps and split for the coupling of specific amino acids as required. This was made possible due to color tagging of the lanterns. Each peptide was separately cleaved using TFA/ $\mathrm{H}_{2} \mathrm{O} / \mathrm{TIS}(95 / 2.5 / 2.5$; v/ v'/ v") for $1 \mathrm{~h}$, concentrated under $\mathrm{N}_{2}$, precipitated in cold $\mathrm{Et}_{2} \mathrm{O}$, washed three times with cold $\mathrm{Et}_{2} \mathrm{O}$ and air-dried overnight. The isolated peptides were analyzed by LCMS and purified by preparative RP-HPLC, leading to the isolation of product with $>95 \%$ purity as assessed by RP-HPLC with yield of 5-20\% (lanterns) and $25-45 \%$ (resins) across all steps.

Formylation, formylated-methionine coupling and acetylation on solid support. Formylation and formylated-methionine coupling were carried out after the final deprotection step as follows: Formic acid or fMet-OH (3 eq) and $\mathrm{HOSu}(3$ eq) were dissolved in DCM at $0{ }^{\circ} \mathrm{C}$ with stirring. $\mathrm{EDC} . \mathrm{HCl}(3 \mathrm{eq})$ was added gradually to the solution before the lanterns were added. DIEA ( 1 eq) was then added to maintain a basic $\mathrm{pH}$; coupling was allowed to proceed overnight at room temperature. Acetylation was carried out in $\mathrm{Ac}_{2} \mathrm{O} / \mathrm{DIEA} / \mathrm{DMF}$ (5:5:90) with shaking overnight following the final deprotection step.

Vancomycin-peptide conjugation. Reactions were performed in $1.5 \mathrm{~mL}$ tubes at $\sim 3 \mathrm{mM}$ concentration. The formylated peptide (1 eq, R1-13, Supplementary Fig. 6) was dissolved in DMF (50 $\mu \mathrm{L} / \mathrm{mg}$ ) and added to azide-functionalized vancomycin (1.1 eq., GPA1-4, Supplementary Fig. 5) dissolved in aqueous sodium ascorbate buffer $(22 \mathrm{mM}, \mathrm{pH} 8.0)^{27}$. Next, an aqueous solution of $\mathrm{CuSO}_{4}(3$ eq., $0.1 \mathrm{M})$ was added, leading to the rapid formation of precipitate. The reaction was monitored by LCMS after shaking for $1 \mathrm{~h}$ and the product purified directly by preparative RP-HPLC, affording $>95 \%$ purity, and overall yield of in $25-47 \%$ (Supplementary Fig. 7).

LC/MS analysis conditions. Analyses were carried out on a Shimadzu HighPerformance Liquid Chromatograph coupled to a Mass Spectrometer LCMS-2020 (ESI, operating both in positive and negative mode) equipped with an SPD-20A Prominence Photo Diode Array Detector and a LC-20AD solvent delivery module running lab solutions v5.97SP1. Analytical separations were performed using a Waters XBridge BEH300 Prep C18 column $(10 \mu \mathrm{m}, 4.6 \times 250 \mathrm{~mm})$. The solvents used were water $+0.1 \%$ formic acid (solvent A) and HPLC-grade ACN $+0.1 \%$ formic acid (solvent B).

Preparative RP-HPLC conditions. Purification using RP-HPLC was performed using a Shimadzu High-Performance Liquid Chromatograph equipped with a SPD-M20A Prominence Photo Diode Array Detector and two LC-20AP pumps running lab solutions v5.97SP1. Preparative separations were performed on a Waters XBridge BEH300 Prep C18 column $(5 \mu \mathrm{m}, 19 \times 150 \mathrm{~mm})$ at a flow rate of $10 \mathrm{~mL} / \mathrm{min}$. The solvents used were water $+0.1 \%$ formic acid (solvent $\mathrm{A}$ ) and HPLC-grade ACN $+0.1 \%$ formic acid (solvent B)
High-resolution mass spectrophotometry analysis of conjugates. Highresolution mass spectrometry measurements were performed on an Orbitrap Fusion mass spectrometer (Thermo Scientific) coupled to a Dionex UltiMate 3000 RSLCnano system equipped with a Dionex UltiMate 3000 RS autosampler, an Acclaim PepMap RSLC analytical column $(75 \mu \mathrm{m}$ x $50 \mathrm{~cm}$, nanoViper, C18, $2 \mu \mathrm{m}$, $100 \AA$ A Thermo Scientific) and an Acclaim PepMap 100 trap column $(100 \mu \mathrm{m}$ x $2 \mathrm{~cm}$, nanoViper, $\mathrm{C} 18,5 \mu \mathrm{m}, 100 \AA$; Thermo Scientific). The peptides were separated by increasing concentrations of $80 \%$ acetonitrile / $0.1 \%$ formic acid at a flow of $250 \mathrm{nl} / \mathrm{min}$ over $30 \mathrm{~min}$. The instrument was operated in alternating datadependent acquisition (DDA) and parallel reaction monitoring (PRM) cycles in such that five ms2 scans were triggered per survey ms1 scan followed by several targeted $\mathrm{ms} 2$ scans to ensure fragmentation of predefined, sample-dependent $\mathrm{m} / \mathrm{z}$ precursors. Each survey msl scan $(300-1800 \mathrm{~m} / \mathrm{z})$ was acquired with a resolution of 240,000 and a normalised AGC (automatic gain control) target of $200 \%$. Dynamic exclusion was set to $10 \mathrm{~s}$ after one occurrence. The five most intense ions were selected for HCD fragmentation (fixed collision energy mode, $24 \% \mathrm{HCD}$ Collision Energy) with a resolution of 15,000, a normalised AGC target of $200 \%$ and a fixed first mass of $100 \mathrm{~m} / \mathrm{z}$. Subsequent targeted $\mathrm{ms} 2$ scans were acquired with essentially identical settings. The raw data files were analysed with QualBrowser (XCalibur 3.0.63, Thermo Scientific) to view spectra and to generate extracted ion chromatograms. The HR-MS data have been deposited to the ProteomeXchange Consortium via the PRIDE partner repository. Data are available via ProteomeXchange with identifier PXD028332.

In silico calculations performed for the fPep library. All formylated peptides were represented in Chemdraw (v19), which was used to calculate the $\log \mathrm{P}$ for each peptide. Additionally, the SMILES codes were used in Elbow-Phoenix (v1.19.2) to perform a simple minimisation and generate their respective.pdb files. Afterwards, the minimised fPeps.pdb were opened in Chimera (v1.15) that allowed the calculation of the total solvent-accessible surface area for each fPeps (Tools/ Surface/ binding analysis/ Measure volume and area).

Bacterial isolates and media. All bacterial isolates used in this study are listed in Supplementary Table 1. The American Type Culture Collection strain (ATCC 29213) and S. aureus strains were cultured onto brain heart infusion (BHI) agar (BD) or into broth (BD) and grown at $37^{\circ} \mathrm{C}$ with shaking. S. aureus was grown in cation adjusted Muller Hinton Broth II (CAMHB, Becton Dickson) for testing. All bacterial strains were stored in glycerol broth at $-80^{\circ} \mathrm{C}$.

Imaging vancomycin binding to $\boldsymbol{S}$. aureus strains. The imaging study was designed to compare the binding interaction to different bacteria by fPep, vancomycin and a fPep-vancomycin conjugate. To minimize the effect of the bulk of the fluorescent dye interfering with vancomycin binding to the bacteria, the fluorescent dye BODIPY was introduced at the C-terminal of vancomycin and fPepvancomycin conjugate. BODIPY ${ }^{\oplus}$ FL NHS Ester (1.1 eq.) dissolved in DMF ( $5 \mathrm{mM}$ ) was added to R11 leading to B1 or propargylamine leading to a BODIPYalkyne compound; the addition of TEA (1 eq.) allowed the completion of the coupling reactions. The labelled vancomycin (B2) and fPep-vancomycin conjugate (B3) were generated using click chemistry between the BODIPY-alkyne and GPA2 for B2 and B1 and GPA2 for B3 (Supplementary Fig. 9).

S. aureus strains (MSSA, ATCC 29213; MRSA, A8090) along with B. subtilis and $E$. coli (DH5 alpha) were grown to mid-exponential phase and incubated with $16 \mu \mathrm{M}$ of the BODIPY-labelled vancomycin (B2); the BODIPY-labelled fPep (B1); or the antibiotic-chemoattractant labelled with BODIPY (B3) for $30 \mathrm{~min}$ at room temperature. The cells were collected by centrifugation $(370 g)$ and washed three times with media to remove the excess unincorporated compound, followed by resuspension in fresh MHB broth. The cells were then imaged using a Zeiss LSM 980 Airyscan 2 (Carl-Zeiss, Jena, Germany) with a 63x oil C-PlanApo 1.4NA objective using the optimal super-resolution mode. Images were processed in FIJI $\mathrm{v}^{36}$ with intensity set across all images. At least three biological replicates were imaged.

Image analysis of BODIPY-labelled vancomycin binding to $S$. aureus strains. $S$. aureus strains A8090 (MRSA), and ATCC 29213 (MSSA) incubated with either fluorescently labeled vancomycin or vancomycin conjugated to fMLFK were analyzed using the FIJ image processing package within Image ${ }^{1}$. Plots of the fluorescence intensity for each cell across the septum and cell walls were determined in triplicate for each cell and the raw data was recorded; more than 120 cells were analyzed per treatment across three biological replicates. The ratios of peak fluorescence values for each cell (wall and septum) were calculated and then aggregated to find the average ratio value for each cell. The aggregated data was visualized using the Graphpad Prism (v9).

Antimicrobial assay. Antimicrobial activity of the antibiotic-chemoattractants was determined as per the Clinical and Laboratory Standards Institute guidelines for microbroth dilution assays. Compounds stocks were prepared as two-fold serial dilution at 10x stock concentration required (final concentration of DMSO 0.5\%). Growth of the S. aureus $\left(5 \times 10^{5} \mathrm{CFU} / \mathrm{mL}\right.$ in CAMHB) was monitored in 96-well flat bottom polypropylene microtiter plates (Greiner) covered with BreatheEasy 
membranes (Diversified Biotech) and absorbance of each well was measured at $595 \mathrm{~nm}$ every $4 \mathrm{~h}$ for $24 \mathrm{~h}$ (Clariostar plate reader, software v5.20R5, BMG). The percentage growth was calculated relative to the no compound control and the average of three biological replicates plotted using GraphPad Prism 9 software. The minimum inhibitory concentration was determined by the lowest drug concentration that inhibited bacterial growth to $90 \%$ compared to the $0 \mu \mathrm{M}$ control $^{63}$.

Neutrophil isolation. For neutrophils used in transwell assays whole blood was collected in the presence of EDTA, under approval from Monash University Human Research Ethics Committee (project \#9572) with written informed consent from healthy human donors; donors were de-identified.

For neutrophils used in microfluidic devices, de-identified fresh blood samples $(10 \mathrm{~mL})$ were purchased from Research Blood Components where blood samples were obtained from healthy volunteers aged $\geq 18$ years who were not receiving immunosuppressant agents. Additionally, venous blood samples from healthy volunteers were collected by phlebotomy, after receipt of written informed consent, and under approval by the MGH Institutional Review Board (protocol 2008-P002123). Neutrophils were isolated from whole blood using EasySep direct human neutrophil isolation kit (StemCell Technologies) as per the manufacturer's instructions.

Transwell chemotaxis assay. The chemotaxis assay was performed in a 96transwell plate with $3 \mu \mathrm{m}$ pores (Corning). The plate was prepared by adding $200 \mu \mathrm{L}$ of peptide in chemotaxis buffer (4 mM L-glutamine, $0.5 \%$ Human Serum Albumin, Sigma, 49\% Roswell Park Memorial Institute (RPMI) 1640, 49\% Hank's buffered salt solution, ThermoFisher) into the bottom receiving wells at a final peptide concentration of $1,10,100$, and $1000 \mathrm{nM}(0.02 \%$ DMSO). The negative control of $0.02 \%$ DMSO was used. The top of the transwell plate was loaded with 200,000 neutrophils in $75 \mu \mathrm{L}$ chemotaxis buffer. The plate was incubated in the dark at $37^{\circ} \mathrm{C}$ with $5 \% \mathrm{CO}_{2}$ for $1.5 \mathrm{~h}$. Neutrophils that had migrated into the bottom receiver plate were lysed by adding $10 \mu \mathrm{L} /$ well of elastase assay buffer (500 mM Tris-HCl, $1 \mathrm{M} \mathrm{NaCl}, \mathrm{pH} 7.4$, and $0.5 \%$ (v/v) Triton X-100), releasing neutrophil elastase. Elastase activity was used as an indicator of neutrophil numbers and was measured by adding the chromogenic elastase substrate N-methoxysuccinyl-Ala-Ala-Pro-Val-p-nitroanilide (Sigma) at a final concentration of 1 $\mathrm{mM}^{35}$. After $30 \mathrm{~min}$ at room temperature, the absorbance at $405 \mathrm{~nm}$ was determined using a microplate reader (Clariostar plate reader, BMG). All peptides were tested in duplicate with 3-4 biological replicates, ensuring different donors each time.

Human neutrophils displayed a variability of response to formylated peptides between individual donors. This variability was taken into account by determining the percentage of neutrophil recruitment relative to the recruitment observed to the fMLFG peptide (FP1) at $100 \mathrm{nM}$ from the same neutrophil donor. To compare data between donors, the positive control of $100 \mathrm{nM} \mathrm{fMLFG} \mathrm{(FP1)} \mathrm{was} \mathrm{set} \mathrm{as} 100 \%$ and all other conditions calculated relative to this. In addition, testing of the entire fPep library or the conjugated library was performed at the same time to allow for comparisons to be made across the results from 3 neutrophil donors. Data were plotted using GraphPad Prism 8 software.

Preparation of microfluidic devices. Microfluidic devices utilized were previously described by Ellett et al. ${ }^{45}$. Briefly, silicon wafers were prepared by sequentially spin-coating two layers with negative photoresist (SU-8, Microchem, Newton, MA), patterning by exposure to UV through two chrome lithography masks (Front Range Photo Mask, Palmer Lake, Colorado), and processing according to manufacturer instructions. The first layer, making up the migration channels, was $5 \mu \mathrm{m}$ thick, while the second layer, making up the outer and inner chambers, was $50 \mu \mathrm{m}$ thick. PDMS (polydimethylsiloxane, Fischer Scientific, Fair Lawn, NJ) devices were fabricated through soft lithography techniques, using the pattered wafer as a negative mold. PDMS base elastomer was mixed thoroughly with curing agent at a ratio of 10:1, poured onto the wafer, and degassed for at least $1 \mathrm{~h}$ in a vacuum chamber. After baking overnight at $75^{\circ} \mathrm{C}$, the cured PDMS was cut from the silicon wafer with a scalpel and the inlets and outlets punched using a $1.0 \mathrm{~mm}$ biopsy punch (Harris Uni-Core ${ }^{\mathrm{TM}}$ ). Individual devices were then cut free using a scalpel, treated with oxygen plasma, and bonded irreversibly to glass-bottom well plates (MatTek, Ashland, MA) by heating to $75^{\circ} \mathrm{C}$ for $10 \mathrm{~min}$.

Use of this microfluidic device for monitoring migration of neutrophils and control of the S. aureus growth in cell culture medium has been outlined in detail previously ${ }^{45}$. Briefly, microfluidic devices were primed with S. aureus (SH1000GFP, $1 \times 10^{6} / \mathrm{mL}$ ) in culture media (Iscove's Modified Dulbecco's Medium $+10 \%$ Fetal Bovine Serum) by loading the outer chamber and applying vacuum to draw bacteria into the inner chambers. The outer chamber was then washed by pushing $200 \mu \mathrm{L}$ of media through the device using a pipette, and loaded with neutrophils $\left(5.5 \times 10^{6} / \mathrm{mL}\right.$, stained with Hoechst 33342 dye, $10 \mathrm{~min}$ at $\left.32 \mu \mathrm{M}\right)$. Additionally, neutrophil phagocytosis was monitored by using pHrodo Red S. aureus BioParticles (Life Technologies) in replace of live S. aureus cells. BioParticles were used at a final concentration of $1 \times 10^{7} / \mathrm{mL}$, which gave 50-100 BioParticles within the microchamber. The concentration of cells were determined by hemocytometer.
Microfluidic data acquisition and analysis. Kinetics of neutrophil migration, $S$. aureus growth and phagocytosis was determined using imaging with an automated Nikon Eclipse TiE inverted wide-field microscope with stage control and perfect focus system run through the Nikon NIS element v4. The biochamber was maintained temperature at $37^{\circ} \mathrm{C}$ and $\mathrm{CO}_{2}$ at $5 \%$ for the $8 \mathrm{~h}$ of the experiment, where images were captured every $5 \mathrm{~min}$ for each field of view (FOV), with at least 4 FOV captured per experimental condition. For each experimental condition at least 12 chambers were tracked for neutrophil migration using the FIJI $^{64}$ plugin Trackmate ${ }^{65}$. The percentage of neutrophils recruited into the chamber was determined relative to the total number of neutrophils in each FOV divided by the number of chambers visible. Data were averaged and the results from at least 3 donors plotted. The pHrodo or S. aureus GFP fluorescence within the microchamber was calculated by thresholding the image and measuring the area in FIJI $^{36}$. Data were plotted using GraphPad Prism 8 software (GraphPad Software, San Diego, CA, USA).

FPR competition binding assay. A competition binding assay was performed based modification of the previous method ${ }^{66}$. To determine the interaction of the formylated peptides with FPR2 of human neutrophils the fluorescently labelled FPR2 antagonist RhB-PB10 was used. RhB-PB10 was synthesized by solid-phase peptide synthesis on Wang resin. After initial acylation with Fmoc-L-Arg(Pbf)-OH, peptide elongation was performed using a solution of 20\% piperidine for Fmoc removal and DIC/ Oxyma for Fmoc-amino acid or Rhodamine B coupling. After completion of the sequence, the peptide was cleaved from the resin and protecting groups were removed via TFA cleavage. Following concentration, the peptide was precipitated using diethyl ether, collected by centrifugation on a spark-resistant centrifuge and purified using RP-HPLC.

RhB-PB10 $(15 \mu \mathrm{M})$ was preincubated with human neutrophils $\left(5 \times 10^{6}\right.$ neutrophils $/ \mathrm{mL}$ ) in Bis-Tris buffer $\mathrm{pH} 6$ for $1 \mathrm{~h}$. Neutrophils were washed twice in Bis-Tris buffer $\mathrm{pH} 6$ to remove any unbound RhB-PB10, before being incubating for 5 min with $8 \mu \mathrm{M}$ of test peptide (fMLFG and fMChaFG both free [FP1 \& FP11] and conjugated [C1 \& C11]) or the control peptides fMLF (Auspep), a specific FPR1 agonist, or fMIVIL (Auspep), an FPR2 specific agonist. Any RhB-PB10 that was competed off by the peptides was then removed by washing in Bis-Tris buffer. Rhodamine B fluorescence levels of each sample were determined using microplate reader (Clariostar plate reader, BMG) with ex $550 \mathrm{~nm}$, em $585 \mathrm{~nm}$.

Measurement of ERK phosphorylation in response to FPR1 or FPR2 activation FlpIn Chinese hamster ovary (CHO) cells stably expressing either the human FPR1 or FPR2. Both of the cell lines were cultured in Dulbecco's modified eagle medium (DMEM), which contained 10\% foetal bovine serum (FBS, JRH Biosciences) and $500 \mu \mathrm{g} / \mathrm{mL}$ of the antibiotic hygromycin-B (Invitrogen Inc). Cells were grown at $37^{\circ} \mathrm{C}$ in a humidified incubator containing $5 \% \mathrm{CO}_{2}$ as described previously ${ }^{67}$. Cells were seeded into 96-well tissue culture plates $\left(4 \times 10^{4}\right.$ cells/well) and after 6-8 h, cells were washed with PBS twice $(50 \mu \mathrm{L} /$ well in each washing step) and then starved by incubating in $90 \mu \mathrm{L} /$ well of serum-free DMEM overnight at $37^{\circ} \mathrm{C}$ in $5 \%$ $\mathrm{CO}_{2}$ before assaying for ERK1/2 phosphorylation (pERK1/2) and intracellular $\mathrm{Ca}^{2+}$ mobilization.

A time course was used to assess peak pERK1/2 phosphorylation for all the compounds. The positive control of $10 \%$ FBS, and serum-free DMEM as vehicle control. FPR specific positive controls were used of FPR1 agonist N-Formyl-MetLeu-Phe (fMLF, Sigma-Aldrich) and the FPR2 agonist, the hexapeptide Trp-LysTyr-Met-Val-DMet (WKYMVm, Tocris Bioscience). All the compounds were tested with time points taken at $0,5,7,10,20,30$. The time versus concentrationresponse curves were generated and the peak-response was observed at $5 \mathrm{~min}$ for all agonists.

For both the time course and concentration-response curves, the stimulation was terminated by the rapid removal of serum-free DMEM with agonists and adding $100 \mu \mathrm{L} /$ well of SureFire lysis buffer. The detection step was performed according to the manufacturer's instructions (AlphaScreen detection kit, PerkinElmer Life and Analytical Science $)^{49}$. Data were normalized to the vehicle (serum-free DMEM) and the response elicited by $10 \%$ FBS, with nonlinear regression performed using GraphPad Prism 9.0.

Measurement of calcium mobilization in response to FPR1 or FPR2 activation For the calcium mobilization assay, the cells were plated and starved in the same manner as the pERK1/2 assay, before washing the cells once in HEPES buffered saline (HBS) solution [150 $\mathrm{mM} \mathrm{NaCl}, 1.3 \mathrm{mM} \mathrm{CaCl}_{2}, 5 \mathrm{mM} \mathrm{KCl}, 1 \mathrm{mM}$ $\mathrm{MgSO}_{4} .7 \mathrm{H}_{2} \mathrm{O}, 1.5 \mathrm{mM} \mathrm{NaHCO} 3,10 \mathrm{mM}$ HEPES, and $10 \mathrm{mM}$ D-glucose; $\mathrm{pH}$ 7.4]. Then the cells were incubated at $37^{\circ} \mathrm{C}$ under $5 \% \mathrm{CO}_{2}$ condition with HBS containing $0.5 \%(\mathrm{w} / \mathrm{v})$ bovine serum albumin (BSA), $4 \mathrm{mM}$ probenecid and $1 \mu \mathrm{M}$ Fluo8-AM (Abcam) for $1 \mathrm{~h}$. FlexStation three plate reader (Molecular Devices) were used to determine $\mathrm{Ca}^{2+}$ mobilization. The peak change in fluorescence signal was normalized to the vehicle (HBS solution) and the cellular response mediated by the positive control, adenosine triphosphate (ATP, $100 \mu \mathrm{M}$ ), and fitted with nonlinear regression curve in GraphPad Prism 9.0.

Quantification of FPR agonist potency and maximal agonist response. Nonlinear regression was performed using GraphPrism 9.0. Concentration-response 
curves mediated by each agonist across the two signaling pathways (intracellular $\mathrm{Ca}^{2+}$ mobilization, pERK1/2 phosphorylation), were fitted to derive the maximal agonist effect $\left(E_{\max }\right)$, normalized to the indicated positive control, and the ligand potency $\left(\mathrm{pEC}_{50}\right)$, defined as the negative logarithm of the agonist concentration that gives a response halfway between the lower and upper asymptotes of the concentration-response. Significance was determined using Student's unpaired $t$ test, with two-tailed $p$ values of $<0.05$ considered statistically significant.

Mouse pneumonia model. All experiments were performed in accordance with the Animal Research Ethics Committee at Monash University (MARP/2018/012). Mice were housed with 12/12 h light-dark cycle with room temperature between 18 and $24^{\circ} \mathrm{C}$, and 40 and $70 \%$ humidity. Eight-week-old female Balb/c mice were anaesthetized with $4 \%$ isoflurane, before intranasal inhalation of $10^{7} \mathrm{CFU}$ S. aureus, in $50 \mu \mathrm{L}$ of $\mathrm{PBS}^{68}$. The concentration of bacteria was important to establish this model in non-neutropenic mice, as too little of an inoculum was easily cleared. In addition, too high of an inoculum with this highly virulent strain resulted in a severe infection reaching protocol determined endpoints within $12 \mathrm{~h}$. At $1 \mathrm{hpi}$ mice were treated intranasally with $50 \mu \mathrm{L}$ of vancomycin $(0.2 \mathrm{mg} / 50 \mu \mathrm{L})$ or equivalent molar amount of fMLFG (FP1), fMChaFG conjugate (C11) or vehicle control (5\% DMSO in PBS). Mice were humanely euthanized by $\mathrm{CO}_{2}$ inhalation at $12 \mathrm{hpi}$ and lung bacterial density was assessed from four mice per treatment group. Lungs were homogenized and underwent serial dilution and plating onto solid media for $\mathrm{CFU}$ enumeration. Significance was determined using a one-way ANOVA with $p<0.05$ being significant and calculated using GraphPad Prism 8 software.

For pathology assessment, excised lungs from one mouse for each of the treatment group was fixed in $10 \%$ buffered formalin before undergoing routine histological processing (Monash University Histology Platform). Lungs were embedded in paraffin, cut into $5 \mu \mathrm{m}$ thick sections and stained with hematoxylin and eosin. Tissue pathology was assessed by a veterinary pathologist (Dr Mark Williamson, Gribbles Veterinary Pathology, Clayton, Australia).

The loss of alveoli structure was quantified in the H\&E sections by determining the area of white space in six FOV $(1.8 \times 1.2 \mathrm{~mm})$ in the outer lobes of the lung (threshold of 0-240). In addition, the number of nuclei in these FOV were also determined. This was performed by isolating nuclei in the image (threshold of $0-130$ ) and then using the analyze particle function (particles $80-4000$, circularity $0.05-1$ ) in $\mathrm{FIJI}^{36}$ to count the number of nuclei detected. Data were plotted using GraphPad Prism 8 software.

Reporting Summary. Further information on research design is available in the Nature Research Reporting Summary linked to this article.

\section{Data availability}

Source data for the figures are provided in the Source Data file. Microscope imaging can be provided on reasonable request to the authors. The high-resolution mass spectrometry data for the compounds have been deposited to the ProteomeXchange Consortium via the PRIDE ${ }^{69}$ partner repository with the dataset identifier PXD028332. Source data are provided with this paper.

Received: 20 May 2020; Accepted: 22 September 2021; Published online: 25 October 2021

\section{References}

1. CDC. Antibiotic resistance threats in the United States. (Report, 2013).

2. O'Neill, J. The Review on antimicrobial resistance. (Report, 2016).

3. Pfaller, M. A., Carvalhaes, C. G., Smith, C. J., Diekema, D. J. \& Castanheira, M. Bacterial and fungal pathogens isolated from patients with bloodstream infection: frequency of occurrence and antimicrobial susceptibility patterns from the SENTRY Antimicrobial Surveillance Program (2012-2017). Diagn. Microbiol. Infect. Dis. 97, 115016 (2020).

4. Guerra, F. E., Borgogna, T. R., Patel, D. M., Sward, E. W. \& Voyich, J. M. Epic immune battles of history: neutrophils vs. Staphylococcus aureus. Front. Cell. Infect. Microbiol. 7, 286 (2017).

5. Jiang, J. H. et al. Antibiotic resistance and host immune evasion in Staphylococcus aureus mediated by a metabolic adaptation. Proc. Natl Acad. Sci. USA 116, 3722-3727 (2019).

6. Howden, B. P., Peleg, A. Y. \& Stinear, T. P. The evolution of vancomycin intermediate Staphylococcus aureus (VISA) and heterogenous-VISA. Infect., Genet. Evolution 21, 575-582 (2014).

7. Ribeiro da Cunha, B., Fonseca, L. P. \& Calado, C. R. C. Antibiotic discovery: Where have we come from, where do we go? Antibiotics (Basel) 8, 45 (2019).

8. Kristian, S. A. et al. Retargeting pre-existing human antibodies to a bacterial pathogen with an alpha-Gal conjugated aptamer. J. Mol. Med. 93, 619-631 (2015).
9. Feigman, M. S. et al. Synthetic immunotherapeutics against Gram-negative pathogens. Cell Chem. Biol. 25, 1185-1194. e5 (2018).

10. Payne, J. A. E. et al. Staphylococcus aureus entanglement in self-assembling $\beta$ peptide nanofibres decorated with vancomycin. Nanoscale Adv. 3, 2607-2616 (2021).

11. Haney, E. F., Straus, S. K. \& Hancock, R. E. W. Reassessing the host defense peptide landscape. Front. Chem. 7, 43 (2019).

12. Hancock, R. E. W., Haney, E. F. \& Gill, E. E. The immunology of host defence peptides: beyond antimicrobial activity. Nat. Rev. Immunol. 16, 321-334 (2016).

13. Dahlgren, C., Gabl, M., Holdfeldt, A., Winther, M. \& Forsman, H. Basic characteristics of the neutrophil receptors that recognize formylated peptides, a danger-associated molecular pattern generated by bacteria and mitochondria. Biochemical Pharmacol. 114, 22-39 (2016).

14. Wen, X. et al. G-protein-coupled formyl peptide receptors play a dual role in neutrophil chemotaxis and bacterial phagocytosis. Mol. Biol. Cell 30, 346-356 (2019).

15. Schiffmann, E., Corcoran, B. A. \& Wahl, S. M. N-formylmethionyl peptides as chemoattractants for leucocytes. Proc. Natl Acad. Sci. USA 72, 1059-1062 (1975).

16. Durr, M. C. et al. Neutrophil chemotaxis by pathogen-associated molecular patterns-formylated peptides are crucial but not the sole neutrophil attractants produced by Staphylococcus aureus. Cell Microbiol. 8, 207-217 (2006).

17. Gao, J. L., Lee, E. J. \& Murphy, P. M. Impaired antibacterial host defense in mice lacking the N-formylpeptide receptor. J. Exp. Med. 189, 657-662 (1999).

18. Fu, H., Dahlgren, C. \& Bylund, J. Subinhibitory concentrations of the deformylase inhibitor actinonin increase bacterial release of neutrophilactivating peptides: a new approach to antimicrobial chemotherapy. Antimicrobial Agents Chemother. 47, 2545-2550 (2003).

19. Butler, M. S., Hansford, K. A., Blaskovich, M. A. T., Halai, R. \& Cooper, M. A. Glycopeptide antibiotics: Back to the future. J. Antibiotics 67, 631-644 (2014).

20. Yim, G., Thaker, M. N., Koteva, K. \& Wright, G. Glycopeptide antibiotic biosynthesis. J. Antibiotics 67, 31-41 (2014).

21. Tsubery, H. et al. Neopeptide antibiotics that function as opsonins and membrane-permeabilizing agents for Gram-negative bacteria. Antimicrobial Agents Chemother. 49, 3122-3128 (2005).

22. Jones, C. N. et al. Bifunctional small molecules enhance neutrophil activities against Aspergillus fumigatus in vivo and in vitro. Front. Immunol. 10, 644 (2019).

23. Marschall, E., Cryle, M. J. \& Tailhades, J. Biological, chemical, and biochemical strategies for modifying glycopeptide antibiotics. J. Biol. Chem. 294, 18769-18783 (2019).

24. Tiyanont, K. et al. Imaging peptidoglycan biosynthesis in Bacillus subtilis with fluorescent antibiotics. Proc. Natl Acad. Sci. 103, 11033-11038 (2006).

25. van Oosten, M. et al. Real-time in vivo imaging of invasive- and biomaterialassociated bacterial infections using fluorescently labelled vancomycin. Nat. Commun. 4, 2584 (2013).

26. Park, H. Y. et al. Comparison of two fluorescent probes in preclinical noninvasive imaging and image-guided debridement surgery of Staphylococcal biofilm implant infections. Sci. Rep. 11, 1622 (2021).

27. Tailhades, J. et al. A chemoenzymatic approach to the synthesis of glycopeptide antibiotic analogues. Angew. Chem. Int. Ed. 59, 10899-10903 (2020).

28. Reynolds, P. E. Structure, biochemistry and mechanism of action of glycopeptide antibiotics. Eur. J. Clin. Microbiol. Infect. Dis. 8, 943-950 (1989).

29. Nagarajan, R. Antibacterial activities and modes of action of vancomycin and related glycopeptides. Antimicrobial Agents Chemother. 35, 605-609 (1991).

30. Gerhard, U., Mackay, J. P., Maplestone, R. A. \& Williams, D. H. The role of the sugar and chlorine substituents in the dimerization of vancomycin antibiotics. Am. Chem. Soc. 115, 232-237 (1993).

31. Yarlagadda, V., Akkapeddi, P., Manjunath, G. B. \& Haldar, J. Membrane active vancomycin analogs: A strategy to combat bacterial resistance. $J$. Medicinal Chem. 57, 4558-4568 (2014).

32. Blaskovich, M. A. T. et al. Protein-inspired antibiotics active against vancomycin- and daptomycin-resistant bacteria. Nat. Commun. 9, 22 (2018).

33. Antonoplis, A. et al. A dual-function antibiotic-transporter conjugate exhibits superior activity in sterilizing MRSA biofilms and killing persister cells. J. Am. Chem. Soc. 140, 16140-16151 (2018).

34. Umstätter, F. et al. Vancomycin resistance is overcome by conjugation of polycationic peptides. Angew. Chem. Int. Ed. 59, 8823-8827 (2020).

35. Okano, A., Isley, N. A. \& Boger, D. L. Peripheral modifications of $[\Psi[\mathrm{CH} 2 \mathrm{NH}] \mathrm{Tpg} 4]$ vancomycin with added synergistic mechanisms of action provide durable and potent antibiotics. Proc. Natl Acad. Sci. 114, E5052-E5061 (2017).

36. Chen, T. et al. Structural basis of ligand binding modes at the human formyl peptide receptor 2. Nat. Commun. 11, 1208 (2020). 
37. Zhuang, Y. et al. Structure of formylpeptide receptor 2-Gi complex reveals insights into ligand recognition and signaling. Nat. Commun. 11, 885 (2020).

38. Freer, R. J. et al. Further studies on the structural requirements for synthetic peptide chemoattractants. Biochemistry 19, 2404-2410 (1980).

39. Dalpiaz, A. et al. Phe-d-Leu-Phe-d-Leu-Phe derivatives as formyl peptide receptor antagonists in human neutrophils: cellular and conformational aspects. BBA - Protein Struct. Mol. Enzymol. 1432, 27-39 (1999).

40. Dalpiaz, A. et al. Met-Ile-Phe-Leu derivatives: full and partial agonists of human neutrophil formylpeptide receptors. Eur. J. Pharmacol. 411, 327-333 (2001).

41. Weiß, E., Schlatterer, K., Beck, C., Peschel, A. \& Kretschmer, D. Formylpeptide receptor activation enhances phagocytosis of community-acquired methicillin-resistant Staphylococcus aureus. J. Infect. Dis. 221, 668-678 (2019).

42. Berend, N., Armour, C. L. \& Black, J. L. Formyl-methionyl-leucylphenylalanine causes bronchoconstriction in rabbits. Agents Actions 17, 466-471 (1986).

43. Peters, M. J., Breslin, A. B., Kemp, A. S., Chu, J. \& Berend, N. Haematological effects of inhalation of $\mathrm{N}$-formyl-methionyl-leucyl-phenylalanine in man. Thorax 47, 284-287 (1992).

44. Clark, R. A., Szot, S., Venkatasubramanian, K. \& Schiffmann, E. Chemotactic factor inactivation by myeloperoxidase-mediated oxidation of methionine. $J$. Immunol. 124, 2020-2026 (1980).

45. Ellett, F. et al. Microfluidic arenas for war games between neutrophils and microbes. Lab on a Chip. 19, 1205-1216 (2019).

46. Ye, R. D. et al. International Union of Basic and Clinical Pharmacology. LXXIII. Nomenclature for the formyl peptide receptor (FPR) family. Pharmacol. Rev. 61, 119-161 (2009).

47. Kretschmer, D., Rautenberg, M., Linke, D. \& Peschel, A. Peptide length and folding state govern the capacity of staphylococcal $\beta$-type phenol-soluble modulins to activate human formyl-peptide receptors 1 or 2. J. Leukoc. Biol. 97, 689-697 (2015).

48. Forsman, H. et al. Structural changes of the ligand and of the receptor alters the receptor preference for neutrophil activating peptides starting with a formylmethionyl group. Biochimica et. Biophysica Acta 1853, 192-200 (2015).

49. Qin, C. X. et al. Small-molecule-biased formyl peptide receptor agonist compound $17 \mathrm{~b}$ protects against myocardial ischaemia-reperfusion injury in mice. Nat. Commun. 8, 14232 (2017)

50. Deora, G. S. et al. Substituted pyridazin-3(2H)-ones as highly potent and biased formyl peptide receptor agonists. J. Medicinal Chem. 62, 5242-5248 (2019).

51. Jiang, D., Xie, T., Liang, J. \& Noble, P. W. $\beta$-Arrestins in the immune system. Prog. Mol. Biol. Transl. Sci. 118, 359-393 (2013).

52. $\mathrm{Fu}$, T. et al. Therapeutic potential of lipoxin A4 in chronic inflammation: focus on cardiometabolic disease. ACS Pharmacol. Transl. Sci. 3, 43-55 (2020).

53. Björnsdottir, H. et al. Phenol-soluble modulin a peptide toxins from aggressive Staphylococcus aureus induce rapid formation of neutrophil extracellular traps through a reactive oxygen species-independent pathway. Front. Immunol. 8, 257 (2017).

54. Qin, C. et al. Cardioprotective potential of annexin-A1 mimetics in myocardial infarction. Pharmacol. Therapeutics 148, 47-65 (2015).

55. Mills, J. S. Differential activation of polymorphisms of the formyl peptide receptor by formyl peptides. Biochimica et. Biophysica Acta 1772, 1085-1092 (2007).

56. Gripentrog, J. M., Mills, J. S., Saari, G. J. \& Miettinen, H. M. Variable responses of formyl peptide receptor haplotypes toward bacterial peptides. Immunogenetics 60, 83-93 (2008).

57. Zhou, C. et al. V101L of human formyl peptide receptor 1 (FPR1) increases receptor affinity and augments the antagonism mediated by cyclosporins. Biochemical J. 451, 245-255 (2013).

58. Otani, T. et al. Polymorphisms of the formylpeptide receptor gene (FPR1) and susceptibility to stomach cancer in 1531 consecutive autopsy cases. Biochemical Biophysical Res. Commun. 405, 356-361 (2011).

59. Maney, P. \& Walters, J. D. Formylpeptide receptor single nucleotide polymorphism $348 \mathrm{~T}>\mathrm{C}$ and its relationship to polymorphonuclear leukocyte chemotaxis in aggressive periodontitis. J. Periodontol. 80, 1498-1505 (2009).

60. Becker, E. L. et al. Broad immunocytochemical localization of the formylpeptide receptor in human organs, tissues, and cells. Cell Tissue Res. 292, 129-135 (1998).

61. Southgate, E. L. et al. Identification of formyl peptides from Listeria monocytogenes and Staphylococcus aureus as potent chemoattractants for mouse neutrophils. J. Immunol. 181, 1429-1437 (2008).

62. Ronga, $\mathrm{L}$. et al. Insertion of multiple $\alpha$-amino $\gamma$-lactam (Agl) residues into a peptide sequence by solid-phase synthesis on synphase lanterns. Pept. Sci. 94, 183-191 (2010).

63. Sun, W. et al. Rapid antimicrobial susceptibility test for identification of new therapeutics and drug combinations against multidrug-resistant bacteria. Emerg. Microbes Infect. 5, 1-11 (2016).
64. Schindelin, J. et al. Fiji: an open-source platform for biological-image analysis. Nat. Methods 9, 676-682 (2012)

65. Tinevez, J.-Y. et al. TrackMate: an open and extensible platform for singleparticle tracking. Methods 115, 80-90 (2017).

66. He, H.-Q., Troksa, E. L., Caltabiano, G., Pardo, L. \& Ye, R. D. Structural determinants for the interaction of formyl peptide receptor 2 with peptide ligands. J. Biol. Chem. 289, 2295-2306 (2014).

67. Corminboeuf, O. \& Leroy, X. FPR2/ALXR agonists and the resolution of inflammation. J. Medicinal Chem. 58, 537-559 (2015).

68. Speir, M. et al. Eliminating Legionella by inhibiting BCL-XL to induce macrophage apoptosis. Nat. Microbiol. 1, 15034 (2016).

69. Perez-Riverol, Y. et al. The PRIDE database and related tools and resources in 2019: improving support for quantification data. Nucleic Acids Res 47, D442-D450 (2019).

70. Medical, B. Medical gallery of Blausen Medical 2014. WikiJ. Med. 1, 10 (2014).

\section{Acknowledgements}

The authors acknowledge the facilities and scientific and technical assistance of the Monash Histology Platform, Department of Anatomy and Developmental Biology, Monash University. Tissue pathology was assessed by a veterinary pathologist Mark Williamson, Gribbles Veterinary Pathology, Clayton, Australia. The authors acknowledge the scientific and technical assistance of the Monash Micro Imaging facility, along with the Monash Animal Research Platform at Monash University, Victoria, Australia. This study used BPA-enabled (Bioplatforms Australia) / NCRIS-enabled (National Collaborative Research Infrastructure Strategy) infrastructure located at the Monash Proteomics and Metabolomics Facility.

Microfabrication of microfluidic devices was conducted at the BioMEMS Resource Center at Massachusetts General Hospital, and supported by a grant from the National Institutes of Health (Grants GM092804 and EB002503) and Shriners Hospital for Children. FE was supported by fellowships from the American Australian Association, Shriners Hospital for Children, and the Executive Committee for Research at the Massachusetts General Hospital.

JAEP was supported by the 2017 Victoria Fellowship from Victorian Endowment for Science, Knowledge and Innovation (VESKI, Australia) and a Hugh Rodgers fellowship from the Melbourne Boston Sister City Association to conduct this research in Boston a Massachusetts General Hospital and Harvard Medical School. MJC is supported by an Australian National Health and Medical Research Council Career Development Fellowship (APP1140619). MJC and JAEP work was supported by Ideas grant from Australian National Health and Medical Research Council (APP2003325). MJC, JAEP and JT are supported by the Australian Research Council Centre of Excellence in Peptide and Protein Science (CE200100012).

AYP is supported by an Australian National Health and Medical Research Council Practitioner Fellowship (APP1117940). GJL was supported by an Australian National Health and Medical Research Council Senior Research Fellowship (APP1044754). The Australian Regenerative Medicine Institute is supported by grants from the State Government of Victoria and the Australian Government. TF supported by a Faculty of Pharmacy and Pharmaceutical Sciences (FPPS) Graduate Research Scholarship and a FPPS International Graduate Research Scholarship provided by Faculty of Pharmacy and Pharmaceutical Sciences, Monash University. While CXQ received a National Heart Foundation Future Leader Fellowship and an Australian National Health and Medical Research Council Grant (APP1187989).

\section{Author contributions}

J.A.E.P. designed and performed experiments for the testing of compounds in neutrophil recruitment, antimicrobial, and in vivo assays. Performed data analysis and wrote the manuscript. J.T. designed and synthesis of the antibiotic-chemoattractants conjugates and fluorescent compounds, along with in silico analysis of fPep library, edited manuscript. F.E. designed and fabricated the microfluidic devices along with designing microfluidic experiments, edited manuscript. X.K. designed and performed the mouse pneumonia model, edited manuscript. A.J.F. performed AiryScan microscopy and data analysis, edited manuscript. T.F. performed F.P.R. activation assays R.L. constructed and tested the fPep library, edited manuscript. A.T. performed image analysis, edited manuscript. S.L. performed microfluidic image analysis and transwell assays, edited manuscript. S.A.W. synthesized BODIPY-labelled compounds, edited the manuscript. R.B.S. performed and analysed HRMS, edited manuscript. G.L. conceptualized experiments to test neutrophil migration and phagocytosis, edited manuscript. C.X.Q. conceptualized F.P.R. experiments and edited manuscript. D.I. designed microfluidic experiments, edited manuscript. A.Y.P. conceptualized experiments and compound design, edited manuscript. M.J.C. developed the concept for the work and assisted in experimental and compound design; writing of the manuscript.

\section{Competing interests}

A.Y.P., J.A.E.P., J.T., and M.J.C. have filed for patent protection (PCT/AU2021/050306) of the antibiotic-chemoattractant compounds. The remaining authors declare no competing interests. 


\section{Additional information}

Supplementary information The online version contains supplementary material available at https://doi.org/10.1038/s41467-021-26244-5.

Correspondence and requests for materials should be addressed to Jennifer A. E. Payne or Max J. Cryle.

Peer review information Nature Communications thanks Huamei Forsman and the other, anonymous, reviewers for their contribution to the peer review of this work. Peer reviewer reports are available.

Reprints and permission information is available at http://www.nature.com/reprints

Publisher's note Springer Nature remains neutral with regard to jurisdictional claims in published maps and institutional affiliations. (c) (1) Open Access This article is licensed under a Creative Commons Attribution 4.0 International License, which permits use, sharing, adaptation, distribution and reproduction in any medium or format, as long as you give appropriate credit to the original author(s) and the source, provide a link to the Creative Commons license, and indicate if changes were made. The images or other third party material in this article are included in the article's Creative Commons license, unless indicated otherwise in a credit line to the material. If material is not included in the article's Creative Commons license and your intended use is not permitted by statutory regulation or exceeds the permitted use, you will need to obtain permission directly from the copyright holder. To view a copy of this license, visit http://creativecommons.org/ licenses/by/4.0/.

(C) The Author(s) 2021 